\title{
Density Functional Approach to Quantumhadrodynamics: Theoretical Foundations and Construction of Extended Thomas-Fermi Models
}

\author{
C. Speicher AND R. M. Dreizler \\ Institut für Theoretische Physik der Universität Frankfurt am Main, Germany \\ AND \\ E. ENGEL \\ University of Toronto, Toronto, Ontario, Canada MSS $1 \mathrm{~A} 7$ \\ Received May 2, 1991; Revised July 25, 1991

\begin{abstract}
We outline the density functional approach to the strong interaction model of quantumhadrodynamics. In particular the extension of the Hohenberg-Kohn theorem to this situation is demonstrated. On the practical level we derive the gradient expansion of the noninteracting kinetic energy to second order in $h^{2}$, including the effects of full four-vector meson exchange as well as vacuum contributions, and discuss the variational equations of the corresponding extended Thomas-Fermi model. (c) 1992 Academic Press, Inc.
\end{abstract}

\section{INTRODUCTION}

Density functional methods have shown to be very successful for the discussion of the quantum many body problem encountered in atomic, molecular, and solid state physics [1]. For the nuclear case it has been demonstrated that these methods are useful for the description of average nuclear groundstate properties in the nonrelativistic regime [2]. The results of Strutinsky-averaged Hartree-Fock (HF) calculations can be reproduced with good accuracy in terms of extended ThomasFermi (TF) models. The most sophisticated applications of these models, using an effective Skyrme-type nucleon-nucleon interaction, include $\hbar$ corrections up to fourth order in the kinetic energy as well as contributions from the effective nucleon mass and the spin-orbit part of the Skyrme potential.

The success of these models on the basis of a nonrelativistic formulation motivates the investigation of density functional methods in the context of relativistic theories of nuclei, such as quantumhadrodynamics (QHD) [3]. This model provides a field dynamical description of hadrons where baryons interact by 
the exchange of mesons. In its simplest version the interaction between the nucleons is mediated by two mesons only, a scalar $\sigma$-meson which is responsible for the attractive force, and a vector $\omega$-meson responsible for the short range repulsion, necessary to reproduce saturation properties. This model, usually referred to as QHD-I, has been extended in several ways. Scalar meson self-interactions of order $\phi^{3}$ and $\phi^{4}$ have been introduced by Boguta and Bodmer [4] where the strengths of these nonlinear interactions are considered as additional, adjustable parameters to be determined by the saturation properties of nuclear matter. For a realistic discussion of nuclei the Coulomb repulsion between the protons must be taken into account as well as an additional isovector particle, the $\rho$-meson, which accounts for the neutron excess in heavy nuclei. The latter extension of the original QHD, referred to as QHD-II, was first considered by Serot [5].

In the present contribution we deal with the simpler case of QHD-I. This model can be solved analytically for nuclear matter in the mean field approximation. First calculations for finite nuclei relied on the TF approximation [6], which extends the nuclear matter results through a local density approximation (LDA) to the inhomogeneous case. Within the $\mathrm{TF}$ approximation nuclear surface properties cannot be reproduced very well. In part, this failure can be traced to the strong inhomogenities in the surface region of the nucleus where the local density approximation fails. This motivated the investigation of inhomogeneity corrections to the LDA. Recently, $\hbar$ corrections of second order to the kinetic energy density have been evaluated for QHD-I by Centelles et al. [7] on the hasis of Wigner transform techniques. The results are restricted to the case of a scalar field $\phi$ and the time-like component $V_{0}$ of the full four-vector field $V_{\mu}$ and involve only valence nuclcons. Dirac sea contributions are neglected.

In the present contribution we extend this calculation in two directions. First we include radiative corrections consistently to order $\hbar^{2}$, i.e., we treat them at the same level as the valence nucleon contributions. In addition we include the spatial components of the four-vector potential in order to account correctly for the baryon current to order $h^{2}$.

Vacuum polarisation effects in QHD have been discussed by Chin [8] for the case of uniform nuclear matter. The nuclear matter results were applied to finite nuclei by Horrowitz and Serot [9] using a LDA for the effective action. Derivative corrections to the effective action and the convergence properties of this series expansion have been assessed by Perry [10]. As the expansion in powers of derivatives of the potentials goes hand in hand with an expansion in inverse powers of the effective mass $M^{*}$, it is claimed that second order derivative terms contribute significantly, while higher order terms, which are at least of order $M^{*-2}$ can be neglected as $M^{*}$ is large compared to the induced energy shifts. These corrections were included in a calculation of finite nuclei within the Hartree approximation by Wasson [11] with the aim of exploring the role of the Dirac sea in nuclei.

Consideration of the full four-potential of the vector meson is of interest if one attempts to describe nuclei, which do not exhibit rotational symmetry. Recently, applications of the mean field limit of QHD to axially symmetric and triaxial nuclei 
have been discussed by several authors $[12,13]$. In particular, it was pointed out by Hofmann and Ring [13] that the magnetic moments of deformed odd A nuclei can only be described successfully with the inclusion of a baryonic polarisation current mediated by the vector meson.

Our derivation of the extended TF formulation of QHD is an extension of previous work for atomic systems on the basis of quantumelectrodynamics (QED) [14]. Apart from the nonzero meson masses it differs (nontrivially) from the atomic case by the presence of an effective position dependent baryon mass introduced by the scalar potential.

In order to set the stage for a wider application of density functional theory (DFT) to QHD we discuss the application of the standard concepts of DFT for this situation. In particular, we proove the extension of the Hohenberg-Kohn theorem, demonstrate how one can extract exchange-correlation contributions in a systematic way in order to prepare a discussion beyond the mean field limit and set up the Kohn-Sham approach to QHD (Section 2). The main body concentrates, however, on the discussion of the extended TF model (to be precise the ThomasFermi-Weizsäcker model), which may be viewed as an approximation of the standard Hartree approach. For this purpose we derive (in Section 3) the gradient expansion of the noninteracting kinetic energy to second order in the derivatives of the scalar and four-current densities. The detailed discussion is initiated by the semiclassical expansion of the propagator of a noninteracting field theory to order $\hbar^{2}$, from which the semiclassical expansion of the scalar density, the four-current density, and the energy density can be extracted (Section 3.1). Renormalisation of divergent vacuum contributions and variants of the normalisation conditions (slightly different from the standard renormalisation scheme) for the low order baryon loop diagrams are discussed in Section 3.2. The final step is the inversion of the semiclassical expansions to obtain the density gradient expansion of the noninteracting kinetic energy functional (Section 3.3). The variational equations of the extended TF model are derived in Section 4. A summary of the results and an indication of possible extensions are found in Section 5.

Throughout the paper we use the units $h=c=1$. For field theoretical quantities we follow the conventions of Ref. [15].

\section{Foundations of a Density Functional Approach to QHD}

In this section we introduce the basic concepts of density functional theory in the context of QHD-I. Extensions to more sophisticated versions of QHD are possible along the lines shown here. The discussion starts with an extension of the existence theorem of Hohenberg and Kohn [16] for the case of a many fermion system interacting by the exchange of massive scalar $(\sigma)$ and vector $(\omega)$ mesons. We then discuss the Kohn-Sham [17] approach for QHD-I and specify the exchangecorrelation contribution to the groundstate energy. 


\subsection{Hohenberg-Kohn theorem for $Q H D$}

The Lagrangian of the scalar-vector meson model has been given by Walecka [3] extending earlier work by Dürr [18]:

$$
\begin{aligned}
\hat{\mathscr{L}}_{\mathrm{QHD}}= & \hat{\psi}\left(i \gamma^{\mu} \partial_{\mu}-\left(M-g_{\mathrm{s}} \hat{\phi}\right)-g_{\mathrm{v}} \gamma^{\mu} \hat{V}_{\mu}\right) \hat{\psi} \\
& +\frac{1}{2}\left(\partial_{\mu} \hat{\phi} \partial^{\mu} \bar{\phi}-m_{\mathrm{s}}^{2} \hat{\phi}^{2}\right)-\frac{1}{4} \bar{F}_{\mu \nu} \hat{F}^{\mu v}+\frac{1}{2} m_{\mathrm{v}}^{2} \hat{V}_{\mu} \hat{V}^{\mu} .
\end{aligned}
$$

Here $\hat{\psi}$ denotes the baryon field operator, $\hat{\phi}$ and $\hat{V}_{\mu}$ the scalar and vector meson field operators, the field tensor is given by

$$
\hat{F}_{\mu v}=\partial_{\mu} \hat{V}_{v}-\partial_{v} \hat{V}_{\mu},
$$

$M$ is the nucleon mass, and $g_{\mathrm{s}}, g_{\mathrm{y}}$ and $m_{\mathrm{s}}, m_{\mathrm{v}}$ are the scalar and vector coupling constants and masses. The vector meson couples minimally to the conserved fourcurrent

$$
j^{\mu}=\hat{\psi} \gamma^{\mu} \hat{\psi}
$$

whereas the scalar meson couples to the scalar density

$$
\hat{\rho}_{\mathrm{s}}=\hat{\psi} \hat{\psi} \hat{\psi}
$$

The extension of the Hohenberg-Kohn theorem [16] to relativistic systems was first considered by Rajagopal and Callaway [19] and by MacDonald and Vosko [20] for the case of QED. In contrast to the standard nonrelativistic situation, for which the basic quantity of the theory is the groundstate density, the covariant relativistic formulation, governed by an external four-potential, has to be based on the four-current density. The Hohenberg-Kohn theorem for QED then states that the groundstate energy of a system of electrons interacting by the exchange of photons and with a classical external four potential is a unique functional of the four current density, $E=E\left[j^{\mu}\right]$. As a consequence of the additional scalar meson interaction, the scalar density has to be included in the context of QHD.

For the proof of the Hohenberg-Kohn theorem, we augment the QHD Hamiltonian derived from Eq. (2.1) by auxiliary, classical external potentials $V_{\text {ext }}^{\mu}$ and $\phi_{\mathrm{ext}}$, to give

$$
\hat{H}=\hat{H}_{\mathrm{QHD}}+\int d^{3} x \hat{\jmath}^{\mu}(x) V_{\mu}^{\mathrm{ext}}(\underline{x})-\int d^{3} x \hat{\rho}_{s}(x) \phi^{\mathrm{ext}}(\underline{x}) .
$$

The potentials are assumed to be independent of time and may be set equal to zero at the end of the argument. As for all field theoretical problems the Hamiltonian (2.4) is not well defined without further prescriptions concerning the elimination of ultraviolet divergencies and divergent vacuum expectation values. Only inclusion of the appropriate counterterm contributions (CTC) in the Lagrangian and subtraction of vacuum expectation values (VEV) (or alternatively normal ordering) 
renders the corresponding groundstate energy finite (see, e.g., Chin [8]). In the following proof we thus use the modified Hamiltonian

$$
\hat{H}_{\mathrm{R}}=\hat{H}+\mathrm{CTC}-\mathrm{VEV}
$$

where the CTC and VEV in the context of the following proof are to be understood as the exact quantities required by renormalisation to all orders.

For a proof of the extension of the Hohenberg-Kohn theorem for QHD we shall work in a suitably defined Schrödinger picture in order to make the connection to nonrelativistic theory more obvious. A detailed discussion of gauge questions and picture dependence of the foundations of DFT is given in [21].

We start with the stationary Schrödinger equation for the groundstate $|g\rangle$,

$$
\hat{H}_{\mathrm{R}}|g\rangle=E|g\rangle,
$$

which defines a map of the set of potentials $\phi_{\mathrm{ext}}$ and $V_{\mathrm{ext}}^{\mu}$ on the set of groundstates $|g\rangle$ (assumed to be nondegenerate) which is surjective by construction. As all fourpotentials that differ only by a gauge transformation of the electrostatic potential $V_{\text {ext }}^{0}$, i.e. $V_{\text {ext }}^{\prime 0}(x)=V_{\text {ext }}^{0}(x)+c$, lead to the same groundstate the map should, more precisely, associate a class of potentials (differing only by these gauge transformations) with a class of groundstates (differing only by global phases).

A second map of all possible groundstates on the groundstate densities $\rho_{\mathrm{s}}$ and $j^{\mu}$ can be established by

$$
\begin{aligned}
\rho_{\mathrm{s}, \mathrm{R}} & =\left\langle g\left|\hat{\rho}_{\mathrm{s}}\right| g\right\rangle+\mathrm{CTC} \\
j_{\mathrm{R}}^{\mu} & =\left\langle g\left|\hat{\jmath}^{\mu}\right| g\right\rangle+\mathrm{CTC},
\end{aligned}
$$

where renormalisation of the densities is implied (of course, the CTC for the renormalisation of $j^{\mu}$ are not identical with those of Eq. (2.5)). Again this map is surjective by construction. We will detail below that this map relates the class of all groundstates deriving from potentials which differ by general time-independent gauge transformations with the corresponding gauge invariant groundstate fourcurrent.

The gist of the proof of the Hohenberg-Kohn theorem is the demonstration that both maps are injective and therefore, as a consequence of a general theorem from the theory of sets, bijective. The proof proceeds by reductio ad absurdum.

In order to demonstrate the injectivity of the first map, one assumes either that $\phi_{\mathrm{ext}}^{\prime}$ is not equal to $\phi_{\mathrm{ext}}$ or that $V_{\mathrm{ext}}^{\prime \mu}$ and $V_{\mathrm{ext}}^{\mu}$ differ by more than a gauge transformation of the electrostatic potential. If the groundstates of the eigenvalue problems with the primed and unprimed potentials

$$
\begin{aligned}
\hat{H}|g\rangle & =E|g\rangle \\
\hat{H}^{\prime}\left|g^{\prime}\right\rangle & =E^{\prime}\left|g^{\prime}\right\rangle
\end{aligned}
$$


were identical (up to global phases $e^{i \alpha}$ ) one would find upon subtraction of both eigenvalue equations

$$
\int d^{3} x\left\{j^{\mu}\left[V_{\mathrm{ext}, \mu}-V_{\mathrm{ext}, \mu}^{\prime}\right]-\hat{\rho}_{s}\left[\phi_{\mathrm{ext}}-\phi_{\mathrm{ext}}^{\prime}\right]\right\}|g\rangle=\left(E-E^{\prime}\right)|g\rangle .
$$

Only for the case explicitly excluded, i.e., $\phi_{\text {ext }}^{\prime}(\underline{x})=\phi_{\text {ext }}(\underline{x})$ and at the same time $V_{\text {ext, }, 0}^{\prime}(\underline{x})=V_{\text {ext, }, 0}(\underline{x})+c, V_{\text {ext, } k}^{\prime}(\underline{x})=V_{\text {ext }, k}(\underline{x})$, would the left hand side of Eq. (2.8) reduce to

$$
-c \int d^{3} x \hat{\jmath}^{0}(\underline{x})|g\rangle=-c Q|g\rangle=\left(E-E^{\prime}\right)|g\rangle
$$

where we have used the fact that the Hamiltonian commutes with the charge operator. Thus both potentials lead to the same groundstate with $E^{\prime}$ reflecting the energy shift introduced by this most simple gauge transformation.

On the other hand one obtains for a general time-independent gauge transformation

$$
V_{\mathrm{ext}, \mu}^{\prime}(\underline{x})=V_{\mathrm{ext}, \mu}(\underline{x})+\partial_{\mu} \Lambda(\underline{x}, t), \quad \Lambda(\underline{x}, t)=c t+\hat{\lambda}(\underline{x})
$$

the relation

$$
-\int d^{3} x\left\{\hat{\jmath}^{i}(\underline{x}) \hat{\partial}_{i} \lambda(\underline{x})+c \hat{\jmath}^{0}(\underline{x})\right\}|g\rangle=\left(E-E^{\prime}\right)|g\rangle .
$$

As $|g\rangle$ is not an eigenstate of $j^{i}(\underline{x})$ this equation leads to a contradiction, showing that the eigenstate $\left|g^{\prime}\right\rangle$ of the general gauge transformed system is no longer identical to $|g\rangle$.

One can also conclude that $|g\rangle$ cannot be an eigenstate of the operator on the left hand side of Eq. (2.8) if the potentials differ by more than a gauge transformation. Consequently the assumption $|g\rangle=\left|g^{\prime}\right\rangle$ again leads to a contradiction. With the convention that potentials differing only by gauge transformations of the electrostatic potential are equivalent, one may state that the map between the classes of equivalent potentials and the corresponding groundstates $|g\rangle$ is injective.

The demonstration of injectivity of the second map also relies on reductio ad absurdum. If one supposes that two groundstates $|g\rangle$ and $\left|g^{\prime}\right\rangle$ which belong to external potentials differing by more than a gauge transformation lead to the same densities $\rho_{\mathrm{s}, \mathrm{R}}$ and $j_{\mathrm{R}}^{\mu}$, then the Raleigh-Ritz principle ${ }^{1}$ states that

$$
\begin{aligned}
E & =\left\langle g\left|\hat{H}_{\mathrm{R}}\right| g\right\rangle\left\langle\left\langle g^{\prime}\left|\hat{H}_{\mathrm{R}}\right| g^{\prime}\right\rangle\right. \\
& =\left\langle g^{\prime}\left|\hat{H}_{\mathrm{R}}^{\prime}\right| g^{\prime}\right\rangle+\left\langle g^{\prime}\left|\hat{H}_{\mathrm{R}}-\hat{H}_{\mathrm{R}}^{\prime}\right| g^{\prime}\right\rangle \\
& =E^{\prime}+\int d^{3} x\left\{j_{\mathrm{R}}^{\mu}\left(V_{\mathrm{ext}, \mu}-V_{\mathrm{ext}, \mu}^{\prime}\right)-\rho_{\mathrm{s}, \mathrm{R}}\left(\phi_{\mathrm{ext}}-\phi_{\mathrm{ext}}^{\prime}\right)\right\} .
\end{aligned}
$$

\footnotetext{
${ }^{1}$ We assume that the Raleigh-Ritz principle is valid for the renormalised Hamiltonian (2.5). To our knowledged a concise proof of the validity of this principle has not been established in a field theoretical context.
} 
It should be noted that the strict inequality, which is crucial for the following argument, does not hold if $|g\rangle$ and $\left|g^{\prime}\right\rangle$ are eigenstates of potentials which are related by a general time-independent gauge transformation, Eq. (2.9). Although the proof of the injectivity of the first map demonstrated that the two states are not identical, this difference is not relevant if one consideres physical observables, i.e., expectation values. Expectation values exhibit only the gauge transformation properties of the operator under consideration, in our case the shift of the energy scale.

Interchanging the roles of primed and unprimed quantities one obtains the statement

$$
E^{\prime}<E-\int d^{3} x\left\{j_{\mathbf{R}}^{\mu}\left(V_{\mathrm{ext}, \mu}-V_{\mathrm{ext}, \mu}^{\prime}\right)-\rho_{\mathrm{s}, \mathrm{R}}\left(\phi_{\mathrm{ext}}-\phi_{\mathrm{ext}}^{\prime}\right)\right\}
$$

Addition of the two inequalities leads to the contradiction

$$
E+E^{\prime}<E+E^{\prime}
$$

Thus the second map is also injective.

Combining the two maps one may state that the class of groundstates which is determined by all potentials differing by a general time-independent gauge transformation is uniquely related to the densities $\rho_{\mathrm{s}}$ and $j^{\mu}$. Any member of this class can thus be considered to be a functional of the densities

$$
|g\rangle=\left|g\left[\rho_{\mathrm{s}}, j^{\mu}\right]\right\rangle
$$

(dropping the index $\mathrm{R}$ for brevity). Of course, the explicit functional form will depend on the specific gauge chosen.

In nonrelativistic DFT the gauge dependence can be exploited by establishing a unique map between the gauge dependent states and the gauge dependent paramagnetic current [22]. In relativistic theory, however, it should be preferable to work with the total current, as physical observables characterized by expectation values

$$
O\left[\rho_{s}, j^{\mu}\right]=\left\langle g\left[\rho_{s}, j^{\mu}\right]|\hat{O}| g\left[\rho_{s}, j^{\mu}\right]\right\rangle
$$

which are unique functionals of the groundstate densities do not depend on the specific gauge chosen.

In particular, insertion of the exact groundstate densities $\rho_{\mathrm{s}}$ and $j^{\mu}$ into

$$
\left\langle g\left[\rho_{\mathrm{s}}, j^{\mu}\right]\left|\hat{H}_{\mathrm{R}}\right| g\left[\rho_{\mathrm{s}}, j^{\mu}\right]\right\rangle
$$

gives the exact groundstate energy $E$ whereas the Raleigh-Ritz principle guarantees that all other $\rho_{\mathrm{s}}^{\prime}, j^{\prime \mu}$ lead to a higher energy,

$$
\left\langle g\left[\rho_{\mathrm{s}}^{\prime}, j^{\prime \mu}\right]\left|\hat{H}_{\mathrm{R}}\right| g\left[\rho_{\mathrm{s}}^{\prime}, j^{\prime \mu}\right]\right\rangle>E .
$$


Ignoring the mathematical subtleties of the $v$-representability problem [23] one may use the unique groundstate energy functional

$$
E\left[\rho_{\mathrm{s}}, j^{\mu}\right] \equiv\left\langle g\left[\rho_{\mathrm{s}}, j^{\mu}\right]\left|\hat{H}_{\mathrm{R}}\right| g\left[\rho_{\mathrm{s}}, j^{\mu}\right]\right\rangle
$$

to obtain the groundstate densities by the variational equations

$$
\begin{aligned}
& \frac{\delta}{\delta \rho_{\mathrm{s}}} E\left[\rho_{\mathrm{s}}, j^{\mu}\right]=0 \\
& \frac{\delta}{\delta j^{\nu}} E\left[\rho_{\mathrm{s}}, j^{\mu}\right]=0
\end{aligned}
$$

which have to be solved under the condition of baryon number and current conservation.

Finally, the functional

$$
F\left[\rho_{\mathrm{s}}, j^{\mu}\right] \equiv E\left[\rho_{\mathrm{s}}, j^{\mu}\right]-\int d^{3} x\left\{j^{\mu}(\underline{x}) V_{\mu}^{\mathrm{ext}}(\underline{x})-\rho_{\mathrm{s}}(\underline{x}) \phi^{\mathrm{ext}}(\underline{x})\right\}
$$

is universal, i.e., it does not depend on the external potentials at all. Note that $F\left[\rho_{\mathrm{s}}, j^{\mu}\right]$ is gauge invariant. It is the external potential energy term

$$
-\int d^{3} x j^{\mu}(\underline{x}) V_{\mu}^{\mathrm{ext}}(\underline{x})
$$

in Eq. (2.12) which produces exactly the required gauge transformation property of the total energy.

This completes the proof of the Hohenberg-Kohn theorem for QHD as we now can set the auxiliary external potentials equal to zero. The functional $F\left[\rho_{\mathrm{s}}, j^{\mu}\right]$ is the energy functional of the QHD system without external potentials.

The proof of the existence of one-to-one mappings between groundstate observables and groundstates densities does not give any hint for the construction of explicit density functionals. This problem still has to be faced. However, the fact that the set of densities may be considered to be the basic variables of the theory makes it wortwhile to pursue this approach to the many body problem.

\subsection{Kohn-Sham Equations for $Q H D$}

For all situations investigated to date, evaluation of the variational approach indicated by Eq. (2.11) or simpler variants leads to results of acceptable rather than the highest possible quality. In order to improve the quality it is necessary to isolate the major physical effects in an accessible form and relegate the more complicated (but hopefully numerically less important) parts into a term that may be accessed step by step. This rearrangement is the aim of the Kohn-Sham scheme [17]. It hinges on the fact that to date no better representation of the kinetic energy functional is available. 
As a first step towards setting up the Kohn-Sham scheme for QHD one defines the kinetic energy $T_{s}$ of a noninteracting system (of nucleons in our case). A noninteracting system in stationary classical external potentials $\phi_{\text {ext }}$ and $V_{\text {ext }}^{\mu}$ is characterized by the Hamiltonian

$$
\hat{H}_{\mathrm{s}}=\int d^{3} x\left\{\hat{\bar{\psi}}\left(-i \gamma^{i} \partial_{i}+M\right) \hat{\psi}+\hat{j}^{\mu}(x) V_{\mu}^{\mathrm{ext}}(\underline{x})-\hat{\rho}_{\mathrm{s}}(x) \phi^{\mathrm{ext}}(\underline{x})\right\},
$$

where renormalisation and subtraction of vacuum expectation values are understood implicitly. The Hohenberg-Kohn theorem then guarantees the existence of the energy functional

$$
E_{\mathrm{s}}\left[\rho_{\mathrm{s}}, j^{\mu}\right] \equiv\left\langle g_{\mathrm{s}}\left[\rho_{\mathrm{s}}, j^{\mu}\right]\left|\hat{H}_{\mathrm{s}}\right| g_{\mathrm{s}}\left[\rho_{\mathrm{s}}, j^{\mu}\right]\right\rangle,
$$

where $\left|g_{\mathrm{s}}\left[\rho_{\mathrm{s}}, j^{\mu}\right]\right\rangle$ is the groundstate of this system. Inserting the Hamiltonian (2.13) into Eq. (2.14) one directly obtains the two parts of this functional, the kinetic energy functional

$$
T_{\mathrm{s}}\left[\rho_{\mathrm{s}}, j^{\mu}\right] \equiv \int d^{3} x\left\langle g_{\mathrm{s}}\left[\rho_{\mathrm{s}}, j^{\mu}\right]\left|\hat{\psi}\left(-i \gamma^{i} \partial_{i}+M\right) \hat{\psi}\right| g_{\mathrm{s}}\left[\rho_{\mathrm{s}}, j^{\mu}\right]\right\rangle
$$

and the obvious external potential term. As $T_{\mathrm{s}}$ is identical with $F$ for a noninteracting system it is universal, i.e., the functional $T_{\mathrm{s}}\left[\rho_{\mathrm{s}}, j^{\mu}\right]$ has the same functional form for all local potentials $\phi_{\mathrm{ext}}$ and $V_{\mathrm{ext}}^{\mu}$.

Next one decomposes the total energy of the interacting system (2.10) in a similar fashion as in nonrelativistic DFT,

$$
\begin{aligned}
E\left[\rho_{\mathrm{s}}, j^{\mu}\right] & =\left\langle g\left[\rho_{\mathrm{s}}, j^{\mu}\right]\left|\hat{H}_{\mathrm{R}}\right| g\left[\rho_{\mathrm{s}}, j^{\mu}\right]\right\rangle \\
& =T_{\mathrm{s}}\left[\rho_{\mathrm{s}}, j^{\mu}\right]+\int d^{3} x\left\{j^{\mu}(\underline{x}) V_{\mu}^{\mathrm{ext}}(\underline{x})-\rho_{\mathrm{s}}(\underline{x}) \phi^{\mathrm{ext}}(\underline{x})\right\}+R\left[\rho_{\mathrm{s}}, j^{\mu}\right],
\end{aligned}
$$

which is simply the definition of the functional $R\left[\rho_{\mathrm{s}}, j^{\mu}\right]$. Although a separation of $R\left[\rho_{\mathrm{s}}, j^{\mu}\right]$ into a Hartree-like term and an exchange-correlation term suggests itself we shall not yet discuss this additional decomposition. The functional derivatives of $R\left[\mu_{\mathrm{s}}, j^{\mu}\right]$ with respect to $\rho_{\mathrm{s}}$ and $j^{\prime \prime}$ lead to local effective potentials depending on the densities. Thus the set of variational equations (2.11) for the interacting system is equivalent to the single particle equation

$$
\left\{-i \underline{\alpha} \cdot \underline{\nabla}+\beta\left[M-\phi^{\mathrm{KS}}(\underline{x})+\gamma^{\mu} V_{\mu}^{\mathrm{KS}}(\underline{x})\right]\right\} \varphi_{i}(\underline{x})=\varepsilon_{i} \varphi_{i}(\underline{x})
$$

with the specific local potentials

$$
\begin{aligned}
& V_{\mu}^{\mathrm{KS}}(\underline{x})=V_{\mu}^{\mathrm{ext}}(\underline{x})+\frac{\delta}{\delta j^{\mu}(\underline{x})} R\left[\rho_{\mathrm{s}}, j^{\mu}\right] \\
& \phi^{\mathrm{KS}}(\underline{x})=\phi^{\mathrm{ext}}(\underline{x})-\frac{\delta}{\delta \rho_{\mathrm{s}}(\underline{x})} R\left[\rho_{\mathrm{s}}, j^{\mu}\right] .
\end{aligned}
$$


Due to the density dependence of the Kohn-Sham potentials Eqs. (2.17) and (2.18) have to be solved selfconsistently together with

$$
\begin{aligned}
& \rho_{\mathrm{s}}(\underline{x})=\frac{1}{2}\left\{\sum_{i, \varepsilon_{i}<\varepsilon_{\mathrm{F}}} \varphi_{i}^{*}(\underline{x}) \gamma^{0} \varphi_{i}(\underline{x})-\sum_{i, \varepsilon_{t}>\varepsilon_{\mathrm{F}}} \varphi_{i}^{*}(\underline{x}) \gamma^{0} \varphi_{i}(\underline{x})\right\} \\
& j^{\mu}(\underline{x})=\frac{1}{2}\left\{\sum_{i, \varepsilon_{i}<\varepsilon_{\mathrm{F}}} \varphi_{i}^{*}(\underline{x}) \gamma^{0} \gamma^{\mu} \varphi_{i}(\underline{x})-\sum_{i, \varepsilon_{i}>\varepsilon_{\mathrm{F}}} \varphi_{i}^{*}(\underline{x}) \gamma^{0} \gamma^{\mu} \varphi_{i}(\underline{x})\right\} .
\end{aligned}
$$

If vacuum polarisation effects are neglected in Eq. (2.19) the sums extend only over the $N$ lowest occupied single particle orbitals with energy eigenvalues $\varepsilon_{i}$ smaller than the Fermi energy $\varepsilon_{\mathrm{F}}$. In a general situation where radiative corrections are included the densities require renormalisation. Before we look more closely at the functional $R\left[\rho_{\mathrm{s}}, j^{\mu}\right]$ it is worthwhile to reiterate the following: The orbitals introduced in the Kohn-Sham approach (as well as the orbital energies) cannot be endowed with a physical meaning (apart from the eigenvalue of the highest occupied orbital). They suffice, however, for the construction of the exact groundstate densities according to Eq. (2.19), provided the functional $R\left[\rho_{s}, j^{\mu}\right]$ is known exactly. The hope is that reasonable approximations for this functional will provide reasonable densities.

\subsection{Specification of $R\left[\rho_{\mathrm{s}}, j^{\mu}\right]$ : Coupling Constant Integration}

For the discussion of $R\left[\rho_{s}, j^{\mu}\right]$ the Hamiltonian has to be specified explicitly on the basis of the Lagrangian (2.1),

$$
\begin{aligned}
\hat{H}= & \hat{H}_{\mathrm{QHD}}+\hat{H}_{\mathrm{ext}} \\
= & \int d^{3} x\left\{\hat{\psi}\left(-i \gamma^{i} \partial_{i}+M\right) \psi+g_{\mathrm{v}} \hat{\psi} \gamma^{\mu} \psi \hat{V}_{\mu}-g_{s} \hat{\psi} \hat{\psi} \hat{\phi}+\hat{\psi} \gamma^{\mu} \hat{\psi} V_{\mathrm{ext}, \mu}-\hat{\bar{\psi}} \hat{\psi} \phi_{\mathrm{ext}}\right. \\
& -\frac{1}{2} \partial_{\mu} \hat{\phi} \partial^{\mu} \hat{\phi}+\left(\hat{\partial}_{0} \hat{\phi}\right)^{2}+\frac{1}{2} m_{s}^{2} \hat{\phi}^{2} \\
& \left.+\frac{1}{2} \partial_{\mu} \hat{V}_{v} \partial^{\mu} \hat{V}^{v}-\partial_{0} \hat{V}_{\mu} \partial^{0} \hat{V}^{\mu}-\frac{1}{2} m_{v}^{2} \hat{V}_{\mu} \hat{V}^{\mu}\right\}
\end{aligned}
$$

We have again included external potentials which will be eliminated in the end. The corresponding field equations are

$$
\begin{aligned}
\left(i \gamma^{\mu} \partial_{\mu}-M\right) \hat{\psi} & =\left(\gamma^{\mu}\left(g_{\mathrm{v}} \hat{V}_{\mu}+V_{\mathrm{ext}, \mu}\right)-\left(g_{\mathrm{s}} \hat{\phi}+\phi_{\mathrm{ext}}\right)\right) \hat{\psi} \\
\left(\partial_{\mu} \partial^{\mu}+m_{\mathrm{s}}^{2}\right) \hat{\phi} & =g_{\mathrm{s}} \hat{\rho}_{\mathrm{s}} \\
\left(\partial_{\mu} \partial^{\mu}+m_{\mathrm{v}}^{2}\right) \hat{V}^{v}-\partial^{v} \partial_{\mu} \hat{V}^{\mu} & =g_{\mathrm{v}} \hat{\jmath}^{v} .
\end{aligned}
$$

The discussion of the Kohn-Sham equations for QHD has demonstrated that there exist local potentials $\phi^{\mathrm{KS}}\left(\left[\rho_{\mathrm{s}}, j^{\mu}\right], \underline{x}\right)$ and $V_{\mu}^{\mathrm{KS}}\left(\left[\rho_{\mathrm{s}}, j^{\mu}\right], \underline{x}\right)$ which produce by solution of Eq. (2.17) the exact groundstate densities in the form (2.19). Thus a noninteracting theory with appropriate external potentials leads to the correct 
densities of the interacting problem. In the following we extend this concept and assume the existence of potentials $\chi_{\mathrm{ext}}^{\lambda}$ and $U_{\mathrm{ext}, \mu}^{\lambda}$ which generate the exact groundstate densities of the interacting system for any given coupling strengths $\lambda g_{\mathrm{s}}$ and $\lambda g_{v}, \lambda \in[0,1]$, i.e., we consider the Hamiltonian

$$
\begin{aligned}
\hat{H}_{\dot{\alpha}}= & \int d^{3} x\left\{\hat{\psi}\left(-i \gamma^{i} \partial_{i}+M\right) \hat{\psi}\right. \\
& +\lambda g_{v} \hat{\psi} \gamma^{\mu} \hat{\psi} \hat{V}_{\mu}-\lambda g_{s} \hat{\psi} \hat{\psi} \hat{\phi}+\hat{\bar{\psi}} \gamma^{\mu} \hat{\psi} U_{\mathrm{ext}, \mu}^{\dot{\lambda}}-\hat{\psi} \hat{\psi} \chi_{\mathrm{ext}}^{2} \\
& -\frac{1}{2} \partial_{\mu} \hat{\phi} \partial^{\mu} \hat{\phi}+\left(\partial_{0} \hat{\phi}\right)^{2}+\frac{1}{2} m_{s}^{2} \hat{\phi}^{2} \\
& \left.+\frac{1}{2} \partial_{\mu} \hat{V}_{v} \partial^{\mu} \hat{V}^{v}-\partial_{0} \hat{V}_{\mu} \partial^{0} \hat{V}^{\mu}-\frac{1}{2} m_{v}^{2} \hat{V}_{\mu} \hat{V}^{\mu}\right\}
\end{aligned}
$$

which again is understood to be renormalised. Clearly, two limiting cases of $\chi_{\mathrm{ext}}^{\hat{2}}$ and $U_{\mathrm{ext}}^{\lambda}$ are known,

$$
\begin{array}{ll}
\chi_{\mathrm{ext}}^{\lambda-1}=\phi_{\mathrm{ext}} & U_{\mathrm{ext}, \mu}^{\lambda=1}=V_{\mathrm{ext}, \mu} \\
\chi_{\mathrm{ext}}^{\lambda=0}=\phi^{\mathrm{KS}} & U_{\mathrm{ext}, \mu}^{\lambda=0}=V_{\mu}^{\mathrm{KS}} .
\end{array}
$$

The corresponding groundstate $\left|g_{i}\right\rangle$ now depends on $\lambda$. On the other hand the scalar and four-current densities are independent of $\lambda$ by construction,

$$
\begin{aligned}
& \rho_{\mathrm{s}}(x)=\left\langle g_{\lambda}\left|\hat{\rho}_{\mathrm{s}}(x)\right| g_{\lambda}\right\rangle \\
& j^{\mu}(x)=\left\langle g_{\lambda}\left|\hat{j}^{\mu}(x)\right| g_{\lambda}\right\rangle .
\end{aligned}
$$

The groundstate energy

$$
E_{\lambda}=\left\langle g_{\lambda}\left|\hat{H}_{\lambda}\right| g_{\lambda}\right\rangle
$$

can be differentiated with respect to $\lambda$ yielding

$$
\begin{aligned}
\frac{d}{d \lambda} E_{\lambda} & =\left\langle g_{\lambda}\left|\frac{d}{d \lambda} \hat{H}_{\lambda}\right| g_{\lambda}\right\rangle \\
& =\int d^{3} x\left\langle g_{\lambda}\left|g_{\mathrm{v}} \hat{\psi} \hat{\gamma}^{\mu} \hat{\psi} \hat{V}_{\mu}-g_{\mathrm{s}} \hat{\psi} \hat{\psi} \hat{\phi}+\hat{\psi} \gamma^{\mu} \hat{\psi} \frac{d}{d \lambda} U_{\mathrm{ext}, \mu}^{\lambda}-\hat{\psi} \hat{\psi} \frac{d}{d \lambda} \chi_{\mathrm{ext}}^{\lambda}\right| g_{\lambda}\right\rangle,
\end{aligned}
$$

where the normalisation $\left\langle g_{\lambda} \mid g_{\lambda}\right\rangle=1$ has been used. Integrating over $\lambda$ leads to

$$
\begin{aligned}
E_{\lambda=1}= & E_{\lambda=0}+\int_{0}^{1} d \lambda \int d^{3} x\left\langle g_{\lambda}\left|g_{\mathrm{v}} \hat{\psi} \gamma^{\mu} \hat{\psi} \hat{V}_{\mu}-g_{\mathrm{s}} \hat{\psi} \hat{\psi} \hat{\phi}\right| g_{\lambda}\right\rangle \\
& +\int d^{3} x\left\{j^{\mu}\left[V_{\mathrm{ext}, \mu}-V_{\mu}^{\mathrm{KS}}\right]-\rho_{\mathrm{s}}\left[\phi_{\mathrm{ext}}-\phi^{\mathrm{KS}}\right]\right\} .
\end{aligned}
$$

Finally one can use the fact that the Kohn-Sham energy $E_{\lambda=0}$ is given by

$$
E_{\hat{\lambda}=0}=T_{\mathrm{s}}+\int d^{3} x\left\{j^{\mu} V_{\mu}^{\mathrm{KS}}-\rho_{\mathrm{s}} \phi^{\mathrm{Ks}}\right\}
$$


as there are no free mesons present in this equivalent noninteracting system. Thus one obtains for the energy of the interacting system

$$
\begin{aligned}
E= & T_{\mathrm{s}}+\int d^{3} x\left\{j^{\mu} V_{\mathrm{ext}, \mu}-\rho_{\mathrm{s}} \phi_{\mathrm{ext}}\right\} \\
& +\int_{0}^{1} d \lambda \int d^{3} x\left\langle g_{\lambda}\left|g_{\nu} \hat{\bar{\psi}} \gamma^{\mu} \hat{\psi} \hat{V}_{\mu}-g_{\mathrm{s}} \hat{\psi} \hat{\psi} \hat{\phi}\right| g_{\lambda}\right\rangle .
\end{aligned}
$$

It is now straightforward to separate a Hartree-like contribution in the last term from the exchange-correlation energy by extracting the disconnected part of the $\lambda$-dependent vertex function,

$$
\begin{aligned}
i G_{\mu, \lambda}^{(2,1,0)}(x, y, z) & =\left\langle g_{\lambda}\left|T \hat{\psi}(x) \hat{\psi}(y) \hat{V}_{\mu}(z)\right| g_{\lambda}\right\rangle \\
& =\lambda g_{v} G_{\lambda}(x, y) \int d^{4} u \operatorname{tr}\left[G_{\lambda}(u, u) \gamma^{v}\right] D_{\mu \nu}^{(0)}(z, u)+i G_{c, \mu, \lambda}^{(2,1,0)}(x, y, z) \\
i G_{\lambda}^{(2,0,1)}(x, y, z) & =\left\langle g_{\lambda}|T \hat{\psi}(x) \hat{\psi}(y) \hat{\phi}(z)| g_{\lambda}\right\rangle \\
& =-\lambda g_{\mathrm{s}} G_{\lambda}(x, y) \int d^{4} u \operatorname{tr}\left[G_{\lambda}(u, u)\right] \Delta^{(0)}(z, u)+i G_{c, \lambda}^{(2,0,0,1)}(x, y, z)
\end{aligned}
$$

Here $G_{\lambda}(x, y)$ is the complete nucleon propagator,

$$
i G_{\lambda}(x, y) \equiv\left\langle g_{\lambda}|T \hat{\psi}(x) \hat{\psi}(y)| g_{\lambda}\right\rangle,
$$

$D_{\mu \nu}^{(0)}(z-u)$ is the noninteracting vector meson, and $\Delta^{(0)}(z-u)$ is the noninteracting scalar meson propagator,

$$
\begin{aligned}
i D_{\mu \nu}^{(0)}(x-y) & \equiv\left\langle g_{\lambda}\left|T \hat{V}_{\mu}(x) \hat{V}_{v}(y)\right| g_{\lambda}\right\rangle \\
& =-i \int \frac{d^{4} k}{(2 \pi)^{4}} e^{-i k(x-y)}\left\{\left(g_{\mu v}-\frac{k_{\mu} k_{v}}{m_{\mathrm{v}}^{2}}\right) \frac{1}{k^{2}-m_{\mathrm{v}}^{2}+i \varepsilon}\right\} \\
i \Delta^{(0)}(x-y) & \equiv\left\langle g_{\lambda}|T \hat{\phi}(x) \hat{\phi}(y)| g_{\lambda}\right\rangle \\
& =i \int \frac{d^{4} k}{(2 \pi)^{4}} e^{-i k(x-y)} \frac{1}{k^{2}-m_{\mathrm{s}}^{2}+i \varepsilon},
\end{aligned}
$$

where we assume that there are no real mesons in the system. The quantities $G_{\mathrm{c}}$ represent the connected part of the vertex functions, i.e., they include only those Feynman diagrams of QHD that do not seperate completely into two parts. Using the fact that by construction the densities are identical for all values of $\lambda$,

$$
\begin{aligned}
& \rho_{\mathrm{s}}(x)=-\operatorname{tr}\left[i G_{\lambda}(x, x)\right] \\
& j^{\mu}(x)=-\operatorname{tr}\left[i \gamma^{\mu} G_{\lambda}(x, x)\right],
\end{aligned}
$$


insertion of Eq. (2.29) into Eq. (2.28) leads to

$$
\begin{aligned}
E= & T_{\mathrm{s}}+\int d^{3} x\left\{j^{\mu}(x) V_{\mathrm{cxt}, \mu}(x)-\rho_{\mathrm{s}}(x) \phi_{\mathrm{ext}}(x)\right\} \\
& +\frac{g_{\mathrm{v}}^{2}}{2} \int d^{3} x d^{4} y j^{\mu}(x) D_{\mu \nu}^{(0)}(x-y) j^{\nu}(y)+\frac{g_{\mathrm{s}}^{2}}{2} \int d^{3} x d^{4} y \rho_{\mathrm{s}}(x) \Delta^{(0)}(x-y) \rho_{\mathrm{s}}(y) \\
& +\int_{0}^{1} d \lambda \int d^{3} x \operatorname{tr}\left[-i g_{\mathrm{v}} \gamma^{\mu} G_{\mathrm{c}, \mu, \lambda}^{(2,1,0)}(x, x, x)+i g_{\mathrm{s}} G_{\mathrm{c}, \lambda}^{(2,0,1)}(x, x, x)\right] .
\end{aligned}
$$

Finally one can rewrite the last term of Eq. (2.31) in terms of the reducible vector $\left(Q_{\mu v}\right)$ and scalar meson $(P)$ polarisation insertions which both are defined to contain only connected diagrams,

$$
\begin{aligned}
E= & T_{\mathrm{s}}+\int d^{3} x\left\{j^{\mu}(x) V_{\mathrm{ext}, \mu}(x)-\rho_{\mathrm{s}}(x) \phi_{\mathrm{ext}}(x)\right\} \\
& +\frac{g_{v}^{2}}{2} \int d^{3} x d^{4} y j^{\mu}(x) D_{\mu \nu}^{(0)}(x-y) j^{\nu}(y)+\frac{g_{\mathrm{s}}^{2}}{2} \int d^{3} x d^{4} y \rho_{\mathrm{s}}(x) \Delta^{(0)}(x-y) \rho_{\mathrm{s}}(y) \\
& +i \int_{0}^{1} \frac{d \lambda}{\lambda} \int d^{3} x d^{4} y\left[D_{\mu \nu}^{(0)}(x-y) Q_{\lambda}^{\mu \nu}(x, y)+\Delta^{(0)}(x-y) P_{\lambda}(x, y)\right]
\end{aligned}
$$

Note that both reducible polarisation insertions include scalar-vector mixing diagrams. Comparing Eq. (2.32) with Eq. (2.16) one immediately identifies $R\left[\rho_{\mathrm{s}}, j^{\mu}\right]$. As expected it consists of the Hartree-like terms in the second line of Eq. (2.32) and an exchange-correlation contribution characterized by the coupling constant integration term,

$$
\begin{aligned}
E_{\mathrm{H}}\left[\rho_{\mathrm{s}}, j^{\mu}\right]= & \frac{g_{\mathrm{v}}^{2}}{2} \int d^{3} x d^{4} y j^{\mu}(x) D_{\mu \nu}^{(0)}(x-y) j^{\nu}(y) \\
& +\frac{g_{\mathrm{s}}^{2}}{2} \int d^{3} x d^{4} y \rho_{\mathrm{s}}(x) \Delta^{(0)}(x-y) \rho_{\mathrm{s}}(y) \\
E_{\mathrm{xc}}\left[\rho_{\mathrm{s}}, j^{\mu}\right]= & i \int_{0}^{1} \frac{d \lambda}{\lambda} \int d^{3} x d^{4} y\left[D_{\mu \nu}^{(0)}(x-y) Q_{\lambda}^{\mu \nu}(x, y)+\Delta^{(0)}(x-y) P_{\lambda}(x, y)\right] .
\end{aligned}
$$

If the system under consideration is translationally invariant, the exchangecorrelation energy density $\varepsilon_{\mathrm{xc}}$ is more easily evaluated in momentum space,

$$
\varepsilon_{\mathrm{xc}}\left[\rho_{\mathrm{s}}, j^{\mu}\right]=i \int_{0}^{1} \frac{d \lambda}{\lambda} \int \frac{d^{4} k}{(2 \pi)^{4}}\left[D_{\mu v}^{(0)}(k) Q_{\lambda}^{\mu \nu}(k)+\Delta^{(0)}(k) P_{\lambda}(k)\right] .
$$

We shall, however, not pursue the discussion of the exchange-correlation energy in 
the present work. Our first aim is the derivation of a gradient expansion of the kinetic energy contribution $T_{\mathrm{s}}$. For a more detailed discussion of the exchangecorrelation contribution in the case of a homogeneous system we refer the reader to the article by Chin [8].

\section{Gradient Expansion of the Noninteracting Kinetic Energy}

The main aim of the present contribution is the derivation of the gradient expansion of the kinetic energy of noninteracting nucleons. For this purpose one bases the considerations on the noninteracting Hamiltonian (2.13). The corresponding Green's function,

$$
i G_{\mathrm{s}}(x, y)=\langle g|T\{\hat{\psi}(x) \hat{\psi}(y)\}| g\rangle,
$$

can, as demonstrated in [24], be expanded in powers of the derivatives of the external potentials, the formal expansion parameter being $\hbar$. In the present case the semiclassical expansion takes the form

$$
G_{\mathrm{s}}=G_{\mathrm{s}}\left[V_{\mu}^{\mathrm{ext}}, \phi^{\mathrm{ext}}, \partial_{i} V_{\mu}^{\mathrm{ext}}, \partial_{i} \phi^{\mathrm{ext}}, \partial_{i} \partial_{j} V_{\mu}^{\mathrm{ext}}, \partial_{i} \partial_{j} \phi^{\mathrm{ext}}, \ldots\right]
$$

Using this expansion one derives directly the corresponding expressions for the scalar and four-current densities,

$$
\begin{aligned}
& \rho_{\mathrm{s}}=\rho_{\mathrm{s}}\left[V_{\mu}^{\mathrm{ext}}, \phi^{\mathrm{ext}}, \partial_{i} V_{\mu}^{\mathrm{ext}}, \partial_{i} \phi^{\mathrm{ext}}, \partial_{i} \partial_{j} V_{\mu}^{\mathrm{ext}}, \partial_{i} \partial_{j} \phi^{\mathrm{ext}}, \ldots\right] \\
& j^{\nu}-j^{v}\left[V_{\mu}^{\mathrm{ext}}, \phi^{\mathrm{ext}}, \partial_{i} V_{\mu}^{\mathrm{ext}}, \partial_{i} \phi^{\mathrm{ext}}, \partial_{i} \partial_{j} V_{\mu}^{\mathrm{ext}}, \partial_{i} \partial_{j} \phi^{\mathrm{ext}}, \ldots\right],
\end{aligned}
$$

and the kinetic energy,

$$
T_{\mathrm{s}}=T_{\mathrm{s}}\left[V_{\mu}^{\mathrm{ext}}, \phi^{\mathrm{ext}}, \partial_{i} V_{\mu}^{\mathrm{ext}}, \partial_{i} \phi^{\mathrm{ext}}, \partial_{i} \partial_{j} V_{\mu}^{\mathrm{ext}}, \partial_{i} \partial_{j} \phi^{\mathrm{ext}}, \ldots\right],
$$

as functionals of the potentials. The details of this first step are outlined in Section 3.1.

The semiclassical functionals require renormalisation, which can be implemented in a straightforward fashion on the basis of the standard renormalisation scheme for relativistic field theories (see, e.g., [25]). The renormalisation procedure is carried through in Section 3.2, where we also indicate a possible alternative to the standard choice of the normalisation conditions.

As a last step the renormalised form of the relations (3.3) must be inverted order by order in $\hbar$, yielding the potentials as approximate functionals of the densities

$$
\begin{aligned}
& \phi^{\mathrm{ext}}=\phi^{\mathrm{ext}}\left[\rho_{\mathrm{s}}, j_{\mu}, \partial_{i} \rho_{\mathrm{s}}, \partial_{i} j_{\mu}, \partial_{i} \partial_{j} \rho_{\mathrm{s}}, \partial_{i} \partial_{j} j_{\mu}, \ldots\right] \\
& V_{v}^{\mathrm{ext}}=V_{v}^{\mathrm{ext}}\left[\rho_{\mathrm{s}}, j_{\mu}, \partial_{i} \rho_{\mathrm{s}}, \partial_{i} j_{\mu}, \partial_{i} \partial_{j} \rho_{\mathrm{s}}, \partial_{i} \partial_{j} j_{\mu}, \ldots\right] .
\end{aligned}
$$


Insertion of these expressions into the renormalised functional (3.4) then leads to the approximate functional

$$
T_{\mathrm{s}}=T_{\mathrm{s}}\left[\rho_{\mathrm{s}}, j_{\mu}, \partial_{i} \rho_{\mathrm{s}}, \partial_{i} j_{\mu}, \partial_{i} \partial_{j} \rho_{\mathrm{s}}, \partial_{i} \partial_{j} j_{\mu}, \ldots\right] .
$$

The approximate functional (3.6) can be applied to set up the extended ThomasFermi (ETF) model as a density functional variant of the Hartree approach to QHD or, with additional statements on the exchange-correlation functional, to extensions of this model.

\subsection{The Noninteracting Propagator}

In a noninteracting ficld theory, the baryon field $\hat{\psi}$ constitutes the only remaining quantised degree of freedom. It is characterized by a Dirac equation with external potentials (the index ext will be dropped for brevity),

$$
\left\{i \gamma^{\mu} \partial_{\mu}-M^{*}(\underline{x})-\gamma^{\mu} V_{\mu}(\underline{x})\right\} \hat{\psi}(x)=0,
$$

where the scalar potential defines the local effective mass

$$
M^{*}(\underline{x}) \equiv M-\phi(\underline{x}) \text {. }
$$

The corresponding noninteracting propagator is the solution of the differential equation

$$
\left\{i \gamma^{\mu} \partial_{\mu}-M^{*}(\underline{x})-\gamma^{\mu} V_{\beta}(\underline{x})\right\} G_{\mathrm{s}}(x, y)=\delta^{4}(x-y) .
$$

Our aim is the expansion of this propagator to order $h^{2}$, that is, to second order in derivatives of the external fields. For the expansion of the propagator we use the method introduced by Engel et al. [24] in which Eq. (3.8) is solved order by order in gradients of the potential leading to an explicit representation of the propagator as a functional of the potentials.

We start with the expansion of $G_{\mathrm{s}}$ in powers of $\hbar$,

$$
G_{\mathrm{s}}(x, y)=\sum_{n=0}^{\infty} G^{[n]}(x, y),
$$

where $G^{[n]}$ is proportional to $h^{n}$ (suppressed here due to our convention $\hbar=1$ ), or equivalently to terms containing $n$ derivatives of the external potentials. Next a phase function is separated from $G^{[n]}$, followed by a Fourier transformation with respect to one of the variables (arbitrarily chosen to be $y$ here),

$$
G^{[n]}(x, y)=e^{-i\left(x^{\nu}-y^{y}\right) V_{v}(x)} \int \frac{d^{4} p}{(2 \pi)^{4}} e^{-i p(x-y)} g^{[n]}(x, p) .
$$

If this ansatz is inserted into Eq. (3.8) one obtains

$$
\delta^{4}(x-y)=e^{-i\left(x^{v}-y^{v}\right) V_{p}(x)} \sum_{n=0}^{\infty} \int \frac{d^{4} p}{(2 \pi)^{4}} e^{-i p(x-y)}\left\{\not p-M^{*}+i \not \partial-i \not \partial V_{v} \frac{\partial}{\partial p_{v}}\right\} g^{[n]}(x, p) .
$$


Collecting terms of the same order in $h$, i.e., in gradients of the potentials, leads to the recursion relation

$$
\left.\left(\not p-M^{*}\right) g^{[n]}(x, p)=\left\{i \not \not V_{v} \frac{\partial}{\partial p_{v}}-\not \not \not\right)\right\} g^{[n-1]}(x, p) \quad n \geqslant 1,
$$

which can be iterated once the lowest order solution $g^{[0]}$ is known. For the case of time independent potentials, it can easily be verified that

$$
\begin{aligned}
g^{[0]}(\underline{x}, p)= & \left(\not p+M^{*}(\underline{x})\right) \\
& \times\left\{\frac{1}{p^{2}-M^{* 2}(\underline{x})+i \varepsilon}+2 \pi i \delta\left(p^{2}-M^{* 2}(\underline{x})\right) \theta\left(p_{0}\right) \theta\left(E_{\mathrm{F}}^{*}(\underline{x})-p_{0}\right)\right\},
\end{aligned}
$$

with the definition of a local Fermi energy

$$
E_{\mathrm{F}}^{*}(\underline{x})=\mu-V_{0}(\underline{x}),
$$

indeed solves Eq. (3.11) in lowest order. ( $\mu$ is the chemical potential of the system.) After multiplication of Eq. (3.12) with $g^{[0]}$ from the left, the recursion can be initiated leading to

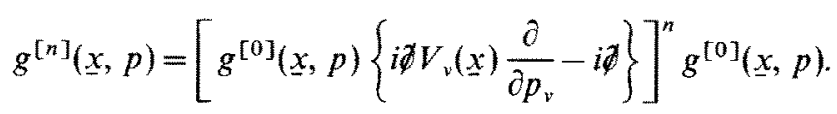

If the propagator $g^{[0]}$ is split into a scalar and a tensor contribution,

$$
g^{[0]}(x, p)=\left(\not p+M^{*}\right) \tilde{g}^{[0]}(\underline{x}, p),
$$

the action of the differential operator in Eq. (3.14) on the scalar part can be expressed as a product of propagators

$$
\left\{i \not V_{v} \frac{\partial}{\partial p_{v}}-i \not\right\} \tilde{g}^{[0]}(\underline{x}, p)=-2 i\left\{p^{v} \not V_{v}+M^{*} \not M^{*}\right\}\left[\tilde{g}^{[0]}(\underline{x}, p)\right]^{2} .
$$

In the Appendix we show that products of $\tilde{g}^{[0]}$ can be decomposed in the form

$$
\left[\tilde{g}^{[0]}(x, p)\right]^{n}=\frac{1}{\left[p_{0}^{2}-E^{* 2}+i \varepsilon\right]^{n}}+2 \pi i \frac{(-1)^{n-1}}{(n-1) !} \frac{\delta^{(n-1)}\left(p^{0}-E^{*}\right)}{\left(p^{0}+E^{*}\right)^{n}} \theta\left(E_{\mathrm{F}}^{*}-p_{0}\right),
$$

where

$$
E^{*}=\sqrt{p^{2}+M^{* 2}}
$$

is the dispersion relation of the effective particle.

Using the algebra of Dirac matrices, the first order contribution to the propagator is found to be 


$$
\begin{aligned}
g^{[1]}(\underline{x}, p)= & -i \partial_{\mu} V_{v}\left\{\left(\not p+M^{*}\right)\left[4 p^{\mu} p^{\nu}\left(\tilde{g}^{[0]}\right)^{3}-\gamma^{\mu} \gamma^{\nu}\left(\tilde{g}^{[0]}\right)^{2}\right]-2 \gamma^{\mu} p^{\nu}\left(\tilde{g}^{[0]}\right)^{2}\right\} \\
& -i \partial_{\mu} M^{*}\left\{\left(\not p+M^{*}\right)\left[4 p^{\mu} M^{*}\left(\tilde{g}^{[0]}\right)^{3}+\gamma^{\mu}\left(\tilde{g}^{[0]}\right)^{2}\right]-2 \gamma^{\mu} M^{*}\left(\tilde{g}^{[0]}\right)^{2}\right\} .
\end{aligned}
$$

The second order contribution can be calculated with a repeat of the same steps to be

$$
\begin{aligned}
& g^{[2]}(\underline{x}, p)=-\partial_{\rho} \partial_{\mu} V_{v}\left\{\left(\not p+M^{*}\right)\left[8 p^{\rho} p^{\mu} p^{\nu}\left(\tilde{g}^{[0]}\right)^{4}-2\left(\gamma^{\mu} \gamma^{v} p^{\rho}+g^{\rho \mu} p^{v}\right)\left(\tilde{g}^{[0]}\right)^{3}\right]\right. \\
& \left.-4 \gamma^{\rho} p^{\mu} p^{v}\left(\tilde{g}^{[0]}\right)^{3}+g^{\rho \mu} \gamma^{v}\left(\tilde{g}^{[0]}\right)^{2}\right\} \\
& -\partial_{\rho} V_{\lambda} \partial_{\mu} V_{v}\left\{( p + M ^ { * } ) \left[48 p^{\rho} p^{\lambda} p^{\mu} p^{\nu}\left(\tilde{g}^{[0]}\right)^{5}+\left(\gamma^{\rho} \gamma^{\lambda} \gamma^{\mu} \gamma^{p}+2 g^{\nu \lambda} g^{\rho \mu}\right)\left(\tilde{g}^{[0]}\right)^{3}\right.\right. \\
& \left.-4\left(3 \gamma^{\rho} \gamma^{\lambda} p^{\mu} p^{v}+2 g^{\rho \mu} p^{\lambda} p^{v}+2 g^{\mu \lambda} p^{\rho} p^{\nu}+2 g^{\nu i} p^{\rho} p^{\mu}\right)\left(\tilde{g}^{[0]}\right)^{4}\right]
\end{aligned}
$$

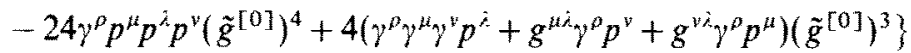

$$
\begin{aligned}
& -\partial_{\rho} \partial_{\mu} M^{*}\left\{\left(\not p+M^{*}\right)\left[8 p^{\rho} p^{\mu} M^{*}\left(\tilde{g}^{[0]}\right)^{4}+2\left(\gamma^{\mu} p^{\rho}-g^{\rho \mu} M^{*}\right)\left(\tilde{g}^{[0]}\right)^{3}\right]\right. \\
& \left.-4 \gamma^{\rho} p^{\mu} M^{*}\left(\tilde{g}^{[0]}\right)^{3}-g^{\rho \mu}\left(\tilde{g}^{[0]}\right)^{2}\right\} \\
& -\partial_{\rho} M^{*} \partial_{\mu} M^{*}\left\{( \not p + M ^ { * } ) \left[48 p^{\rho} p^{\mu} M^{* 2}\left(\tilde{g}^{[0]}\right)^{5}-g^{\rho \mu}\left(\tilde{g}^{[0]}\right)^{3}\right.\right. \\
& \left.+4\left(2 \gamma^{\mu} p^{\rho} M^{*}+\gamma^{\rho} p^{\mu} M^{*}+2 p^{\rho} p^{\mu}-2 g^{\rho \mu} M^{* 2}\right)\left(\tilde{g}^{[0]}\right)^{4}\right] \\
& \left.-24 \gamma^{\rho} p^{\mu} M^{* 2}\left(\tilde{g}^{[0]}\right)^{4}-4\left(g^{\rho \mu} M^{*}+\gamma^{\beta} p^{\mu}\right)\left(\tilde{g}^{[0]}\right)^{3}\right\} \\
& -\partial_{\rho} M^{*} \partial_{\mu} V_{v}\left\{( \not p + M ^ { * } ) \left[96 p^{\rho} p^{\mu} p^{\nu} M^{*}\left(\tilde{g}^{[0]}\right)^{5}-\left(\gamma^{\rho} \gamma^{\mu} \gamma^{\nu}+\gamma^{\mu} \gamma^{v} \gamma^{\rho}\right)\left(\tilde{g}^{[0]}\right)^{3}\right.\right. \\
& \left.-4\left(3 \gamma^{\mu} \gamma^{v} p^{\rho} M^{*}+4 g^{\rho \mu} p^{v} M^{*}+2 g^{\rho v} p^{\mu} M^{*}-3 \gamma^{\rho} p^{\mu} p^{v}\right)\left(\tilde{g}^{[0]}\right)^{4}\right] \\
& -24\left(\gamma^{\beta} p^{\mu} p^{\nu} M^{*}+\gamma^{\mu} p^{\rho} p^{v} M^{*}\right)\left(\tilde{g}^{[0]}\right)^{4} \\
& \left.+4\left(\gamma^{\rho} \gamma^{\mu} \gamma^{v} M^{*}+g^{\rho v} \gamma^{\mu} M^{*}-\gamma^{\mu} \gamma^{\rho} p^{v}\right)\left(\tilde{g}^{[0]}\right)^{3}\right\} .
\end{aligned}
$$

Vacuum and valence nucleon parts can be seperated by use of Eq. (3.17). In the limit $M^{*}=M$ this expression reduces to the result of Engel et al. [24].

It is worthwhile to note at this point that the ansatz (3.10) is not the only possibility to initiate a gradient expansion. A more symmetrical formulation would arise if one were to use center of mass and relative coordinates in conjunction with a Wigner transformation of the propagator

$$
G^{[n]}(R, s)=e^{-i s^{v} V_{v}[R]} \int \frac{d^{4} p}{(2 \pi)^{4}} e^{-i p s} g_{\mathrm{W}}^{[n]}(R, p)
$$

with $R=\frac{1}{2}(x+y)$ and $s=x-y$.

On the basis of this equation a Wigner-Kirkwood expansion of $g_{\mathrm{w}}(R, p)$ could be generated after the Dyson equation was transformed into phase space. Though the Wigner-Kirkwood expansion is related to the expansion based on the Fourier transformation, there is no simple relation between $g^{[n]}(x, p)$ and $g_{\mathrm{W}}^{[n]}(R, p)$, as the arrangement of $h$ contributions in the two series is different. Relevant remarks on 
this point (though for the case of the Bloch density matrix rather than the Green's function) can be gleaned from [26].

With the knowledge of the propagator the calculation of expectation values of interest is straightforward. For the vacuum part we use dimensional regularisation [27] to handle the divergent expressions. Renormalisation is necessary to extract the finite contributions. All expressions are given for the case of pure neutron matter. Therefore, the spin-isospin degeneracy factor has the value 2 , whereas it would have the value 4 for nuclear matter. With the definition of a local Fermi momentum

$$
k_{\mathrm{F}}(\underline{x})=\sqrt{E_{\mathrm{F}}^{* 2}(\underline{x})-M^{* 2}(\underline{x})} \theta\left(E_{\mathrm{F}}^{* 2}(\underline{x})-M^{* 2}(\underline{x})\right),
$$

the four-current, split into a regularised vacuum (reg) and a valence nucleon (D) part, to second order is

$$
\begin{aligned}
j^{\alpha}(\underline{x})= & -i \lim _{y \rightarrow x} \operatorname{tr}\left\{\gamma^{\alpha} G_{\mathrm{s}}(x, y)\right\}=j_{\mathrm{reg}}^{\alpha}(\underline{x})+j_{D}^{\alpha}(\underline{x}) \\
j_{\mathrm{reg}}^{\alpha}= & -\frac{1}{12 \pi^{2}} \partial_{i}\left[F^{i x}\left(\Gamma\left(2-\frac{D}{2}\right)-2 \ln \left|\frac{M^{*}}{m}\right|\right)\right] \\
\rho_{\mathrm{D}}= & \frac{1}{3 \pi^{2}} k_{\mathrm{F}}^{3}-\frac{1}{12 \pi^{2}}\left(\frac{E_{\mathrm{F}}^{*}}{k_{\mathrm{F}}}+2 \ln \frac{k_{\mathrm{F}}+E_{\mathrm{F}}^{*}}{M^{*}}\right) \Delta V_{0} \\
& +\frac{1}{24 \pi^{2}} \frac{1}{k_{\mathrm{F}}}\left(3-\frac{E_{\mathrm{F}}^{* 2}}{k_{\mathrm{F}}^{2}}\right)\left(\underline{\nabla} V_{0}\right)^{2}+\frac{1}{24 \pi^{2}} \frac{1}{k_{\mathrm{F}}} F_{i j} F^{i j}+\frac{1}{12 \pi^{2}} \frac{M^{*}}{k_{\mathrm{F}}} \Delta \phi \\
& +\frac{1}{24 \pi^{2}} \frac{1}{k_{\mathrm{F}}}\left(2-\frac{E_{\mathrm{F}}^{* 2}}{k_{\mathrm{F}}^{2}}\right)(\underline{\nabla} \phi)^{2}-\frac{1}{12 \pi^{2}} \frac{1}{M^{*}} \frac{E_{\mathrm{F}}^{*}}{k_{\mathrm{F}}}\left(3-\frac{E_{\mathrm{F}}^{* 2}}{k_{\mathrm{F}}^{2}}\right)\left(\underline{\nabla} V_{0}\right)(\underline{\nabla} \phi) \\
j_{D}^{k}= & \frac{1}{6 \pi^{2}} \partial_{i}\left[F^{i k} \ln \frac{k_{\mathrm{F}}+E_{\mathrm{F}}^{*}}{M^{*}}\right],
\end{aligned}
$$

where $m$ is the mass scale introduced by dimensional regularisation, $D$ is the dimension of the Minkowski space in which the integration has been performed, and $F_{i j}=\partial_{i} V_{j}-\partial_{j} V_{i}$. The condition of current conservation is automatically satisfied.

The second basic quantity in QHD is the scalar density

$$
\begin{aligned}
\rho_{\mathrm{s}}(\underline{x})= & -i \lim _{y \rightarrow x} \operatorname{tr}\left\{G_{\mathrm{s}}(x, y)\right\}=\rho_{\mathrm{s}, \mathrm{reg}}(\underline{x})+\rho_{\mathrm{s}, \mathrm{D}}(\underline{x}) \\
\rho_{\mathrm{s}, \mathrm{reg}}= & +\frac{1}{4 \pi^{2}} M^{* 3}\left[\Gamma\left(2-\frac{D}{2}\right)+1-2 \ln \left|\frac{M^{*}}{m}\right|\right] \\
& +\frac{1}{12 \pi^{2}} \frac{1}{M^{*}}\left(\underline{\nabla} V_{0}\right)^{2}-\frac{1}{24 \pi^{2}} \frac{1}{M^{*}} F_{i j} F^{i j}+\frac{1}{8 \pi^{2}} \frac{1}{M^{*}}(\underline{\nabla} \phi)^{2} \\
& +\frac{1}{4 \pi^{2}}\left[\frac{1}{2} \Gamma\left(2-\frac{D}{2}\right)-\frac{1}{3}-\ln \left|\frac{M^{*}}{m}\right|\right] \Delta \phi
\end{aligned}
$$




$$
\begin{aligned}
\rho_{\mathrm{s}, \mathrm{D}}= & \frac{1}{2 \pi^{2}} M^{*}\left(k_{\mathrm{F}} E_{\mathrm{F}}^{*}-M^{* 2} \ln \frac{k_{\mathrm{F}}+E_{\mathrm{F}}^{*}}{M^{*}}\right) \\
& -\frac{1}{12 \pi^{2}} \frac{M^{*}}{k_{\mathrm{F}}} \Delta V_{0}-\frac{1}{24 \pi^{2}} \frac{1}{M^{*}} \frac{E_{\mathrm{F}}^{*}}{k_{\mathrm{F}}}\left(1+\frac{E_{\mathrm{F}}^{* 2}}{k_{\mathrm{F}}^{2}}\right)\left(\nabla V_{0}\right)^{2} \\
& +\frac{1}{24 \pi^{2}} \frac{1}{M^{*}} \frac{E_{\mathrm{F}}^{*}}{k_{\mathrm{F}}} F_{i j} F^{i j}+\frac{1}{12 \pi^{2}}\left(\frac{E_{\mathrm{F}}^{*}}{k_{\mathrm{F}}}-3 \ln \frac{k_{\mathrm{F}}+E_{\mathrm{F}}^{*}}{M^{*}}\right) \Delta \phi \\
& -\frac{1}{24 \pi^{2}} \frac{1}{M^{*}} \frac{E_{\mathrm{F}}^{*}}{k_{\mathrm{F}}}\left(2+\frac{E_{\mathrm{F}}^{* 2}}{k_{\mathrm{F}}^{2}}\right)(\nabla \phi)^{2} \\
& +\frac{1}{12 \pi^{2}} \frac{1}{k_{\mathrm{F}}}\left(2+\frac{E_{\mathrm{F}}^{* 2}}{k_{\mathrm{F}}^{2}}\right)\left(\nabla V_{0}\right)(\nabla \phi) .
\end{aligned}
$$

In contrast to the four-current, the scalar density is divergent even in lowest order. The total baryonic energy density of the noninteracting system is given by

$$
\begin{aligned}
\varepsilon^{\mathrm{B}}(\underline{x})= & \lim _{y \rightarrow x} \operatorname{tr}\left\{\gamma^{0} \partial_{x}^{0} G_{\mathrm{s}}(x, y)\right\}=\varepsilon_{\mathrm{reg}}^{\mathrm{B}}(\underline{x})+\varepsilon_{\mathrm{D}}^{\mathrm{B}}(\underline{x}) \\
\varepsilon_{\mathrm{reg}}^{\mathrm{B}}= & \rho_{\mathrm{reg}} V_{0}+\frac{1}{8 \pi^{2}} M^{* 4}\left[\frac{1}{2} \Gamma\left(2-\frac{D}{2}\right)+\frac{3}{4}-\ln \left|\frac{M^{*}}{m}\right|\right] \\
& +\frac{1}{24 \pi^{2}}\left[\Gamma\left(2-\frac{D}{2}\right)-2 \ln \left|\frac{M^{*}}{m}\right|\right]\left(\left(\underline{\nabla} V_{0}\right)^{2}+\frac{1}{2} F_{i j} F^{i j}\right) \\
& +\frac{1}{8 \pi^{2}}\left[\frac{1}{2} \Gamma\left(2-\frac{D}{2}\right)-\frac{1}{3}-\ln \left|\frac{M^{*}}{m}\right|\right](\nabla \phi)^{2} \\
\varepsilon_{\mathrm{D}}^{\mathrm{B}}= & \rho_{\mathrm{D}} V_{0}+\frac{1}{8 \pi^{2}}\left(k_{\mathrm{F}} E_{\mathrm{F}}^{* 3}+k_{\mathrm{F}}^{3} E_{\mathrm{F}}^{*}-M^{* 4} \ln \frac{k_{\mathrm{F}}+E_{\mathrm{F}}^{*}}{M^{*}}\right) \\
& -\frac{1}{12 \pi^{2}} k_{\mathrm{F}}\left(1+\frac{E_{\mathrm{F}}^{* 2}}{k_{\mathrm{F}}^{2}}\right) \Delta V_{\mathrm{o}}+\frac{1}{12 \pi^{2}}\left(\frac{E_{\mathrm{F}}^{*}}{k_{\mathrm{F}}}-\frac{1}{2} \frac{E_{\mathrm{F}}^{* 3}}{k_{\mathrm{F}}^{3}}-\ln \frac{k_{\mathrm{F}}+E_{\mathrm{F}}^{*}}{M^{*}}\right)\left(\nabla V_{0}\right)^{2} \\
& +\frac{1}{24 \pi^{2}}\left(\frac{E_{\mathrm{F}}^{*}}{k_{\mathrm{F}}}-\ln \frac{k_{\mathrm{F}}+E_{\mathrm{F}}^{*}}{M^{*}}\right) F_{y} F^{i j}+\frac{1}{12 \pi^{2}} M^{*}\left(\frac{E_{\mathrm{F}}^{*}}{k_{\mathrm{F}}}-\ln \frac{k_{\mathrm{F}}+E_{\mathrm{F}}^{*}}{M^{*}}\right) \Delta \phi \\
& +\frac{1}{24 \pi^{2}}\left(\frac{E_{\mathrm{F}}^{*}}{k_{\mathrm{F}}}-\frac{E_{\mathrm{F}}^{* 3}}{k_{\mathrm{F}}^{3}}-\ln \frac{k_{\mathrm{F}}+E_{\mathrm{F}}^{*}}{M^{*}}\right)(\underline{\nabla} \phi)^{2} \\
& -\frac{1}{12 \pi^{2}} \frac{M^{*}}{k_{\mathrm{F}}}\left(1-\frac{E_{\mathrm{F}}^{* 2}}{k_{\mathrm{F}}^{2}}\right)\left(\underline{\nabla}_{0}\right)(\underline{\nabla} \phi) .
\end{aligned}
$$

To derivc the represcntation of the vacuum contribution in Eq. (3.23), a partial integration has been carried out to eliminate a term $\sim \Delta \phi$. 
The consistency of the results (3.21) (3.23) on the semiclassical level can be checked using the relations

$$
\begin{aligned}
& \frac{\delta}{\delta V_{v}} \int d^{3} x\left\{\varepsilon^{\mathrm{B}}(\underline{x})-\mu j^{0}(\underline{x})\right\}=j^{v} \\
& \frac{\delta}{\delta \phi} \int d^{3} x\left\{\varepsilon^{\mathrm{B}}(\underline{x})-\mu j^{0}(\underline{x})\right\}=-\rho_{\mathrm{s}} .
\end{aligned}
$$

These relations are easily derived by a minimisation of the functional (2.14) using the fact that $T_{\mathrm{s}}$, Eq. (2.15), does not depend on the external potentials and the subsidiary condition of a fixed particle number to render the minimisation meaningful.

\subsection{Renormalisation}

The semiclassical results (3.21), (3.22) for the four current and the scalar density of a noninteracting system (indicatcd diagrammatically in Figs. 1(a, b) as well as for the total energy (3.23) display the ultraviolet divergencies of field theory in the approximation applied. In order to obtain meaningful expressions for the semiclassical functionals renormalisation has to be carried through. For the discussion of renormalisation it is sufficient to consider the vacuum expectation values of the system. All other groundstate expectation values are automatically rendered finite by the counterterms introduced for the vacuum.

In an effective field theory describing a system of noninteracting fermions in external four- and scalar potentials the only sources for divergencies are closed fermion loops. The number of external vector or scalar potentials attached determines whether a given diagrammatic contribution is convergent or divergent. In particular, the renormalisation of the scalar and vector parts decouples completely in this situation.

With power counting and use of Furry's theorem as well as gauge invariance one can identify the five divergent diagrams of Fig. 2. Defining the irreducible $(m, n)$ point functions $\Gamma^{(m, n)}$ of the interacting theory as

$$
\begin{aligned}
& \Gamma_{\mu_{1} \ldots \mu_{m}}^{(m, n)}\left(x_{1}, \ldots, x_{m} ; y_{1}, \ldots, y_{n}\right) \\
& \equiv\left(-i g_{\mathrm{v}}\right)^{m}\left(i g_{\mathrm{s}}\right)^{n}<0 \mid T \psi\left(x_{1}\right) \gamma_{\mu_{1}} \psi\left(x_{1}\right) \ldots \\
& \quad \psi\left(x_{m}\right) \gamma_{\mu_{m}} \psi\left(x_{m}\right) \psi\left(y_{1}\right) \psi\left(y_{1}\right) \ldots \psi\left(y_{n}\right) \psi\left(y_{n}\right)|0>l|_{\text {irred }}
\end{aligned}
$$

where $m$ denotes the number of external vector, $n$ the number of external scalar vertices and the index "irred" indicates that only irreducible diagrams have to be included, onc recognises that the fermion loops in Fig. 2 are based on the lowest order, that is the noninteracting, contributions to the irreducible $(m, n)$-point functions, in particular $\Gamma^{(2,0)^{(0)}}$ (Fig. 2a) and $\Gamma^{(0.1)^{(0)}}$ to $\Gamma^{(0,4)^{(0)}}$ (Fig. 2(b-e)). As Furry's theorem extends to mixtures of vector and scalar vertices one can write the 
a

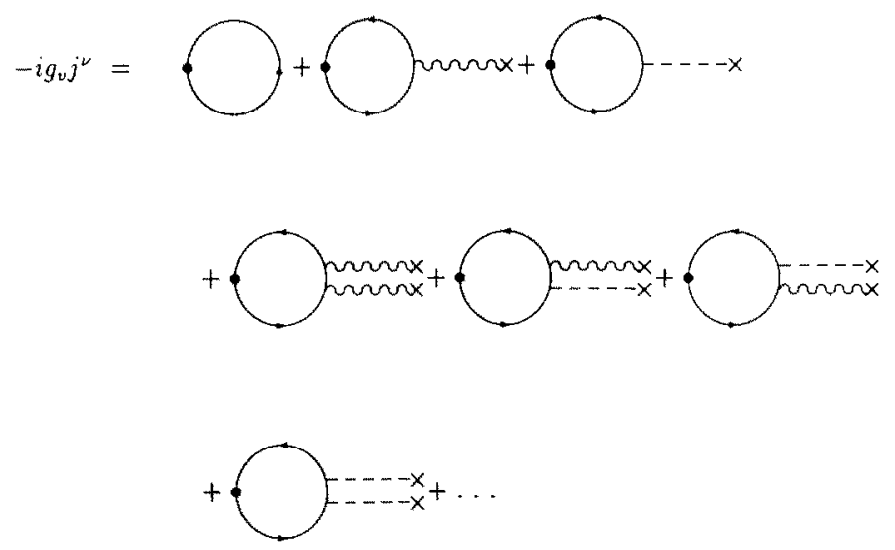

b

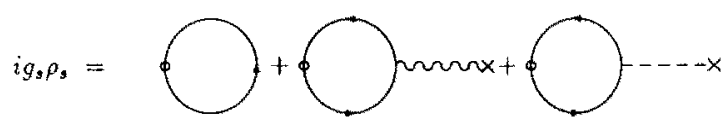
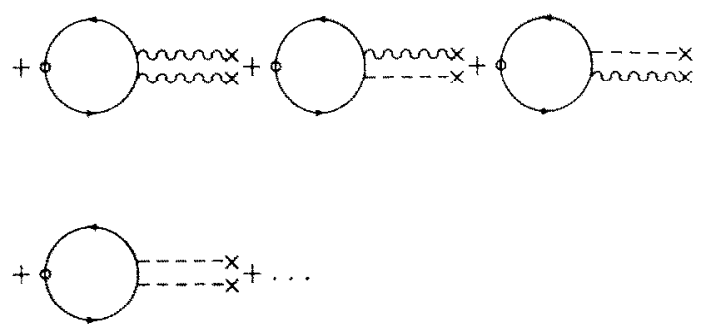

FIG. 1. Ground state four-current (a) and scalar density (b) of the noninteracting system: $=-i g_{\mathrm{v}} \gamma^{v}, \quad=i g_{\mathrm{s}}, \quad \sim m x=-i g_{\mathrm{v}} \gamma_{\mathrm{v}} V_{\mathrm{ext}}^{v},-\cdots--\mathrm{x}=i g_{\mathrm{s}} \Phi_{\mathrm{ext}}$, and $\rightarrow=$ ground state nucleon propagator.

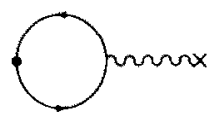

(a)

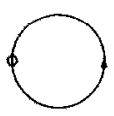

(b)

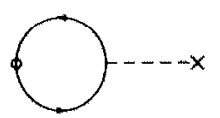

(c)

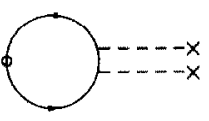

(d)

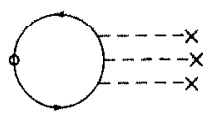

(e)

FIG. 2. Divergent graphs contributing to the vacuum four-current and scalar density of the noninteracting system $-=-i g_{v} \gamma^{\nu}, O=i g_{s}, \sim m x=-i g_{v} \gamma_{v} V_{\text {ext }}^{\nu},---x=i g_{s} \Phi_{\text {ext }}$, and $\rightarrow=$ vacuum nucleon propagator. 
vacuum contribution to the four-current and scalar densities of the noninteracting system as

$$
\begin{aligned}
& -i g_{v} j_{v}(z)=\int d^{4} x \Gamma_{v \mu}^{(2,0)(0)}(z, x) V^{\mu}(x) \\
& +\int d^{4} x d^{4} y \Gamma_{v \mu}^{(2,1)^{(0)}}(z, x ; y) V^{\mu}(x) \phi(y) \\
& +\frac{1}{2} \int d^{4} x d^{4} y_{1} d^{4} y_{2} \Gamma_{v \mu}^{(2,2)^{(0)}}\left(z, x ; y_{1}, y_{2}\right) V^{\mu}(x) \phi\left(y_{1}\right) \phi\left(y_{2}\right) \\
& +\frac{1}{6} \int d^{4} x_{1} d^{4} x_{2} d^{4} x_{3} \Gamma_{\nu \mu_{1} \mu_{2} \mu_{3}}^{(4,0)}\left(z, x_{1}, x_{2}, x_{3}\right) V^{\mu_{1}}\left(x_{1}\right) V^{\mu_{2}}\left(x_{2}\right) V^{\mu_{3}}\left(x_{3}\right) \\
& +\cdots \\
& i g_{\mathrm{s}} \rho_{\mathrm{s}}(z)=\Gamma^{(0,1)^{(0)}}(z)+\int d^{4} y \Gamma^{(0,2)^{(0)}}(z, y) \phi(y) \\
& +\frac{1}{2} \int d^{4} y_{1} d^{4} y_{2} \Gamma^{(0.3)^{(0)}}\left(z, y_{1}, y_{2}\right) \phi\left(y_{1}\right) \phi\left(y_{2}\right) \\
& +\frac{1}{6} \int d^{4} y_{1} d^{4} y_{2} d^{4} y_{3} \Gamma^{(0,4)(0 \mid}\left(z, y_{1}, y_{2}, y_{3}\right) \phi\left(y_{1}\right) \phi\left(y_{2}\right) \phi\left(y_{3}\right) \\
& +\frac{1}{2} \int d^{4} x_{1} d^{4} x_{2} \Gamma_{\mu_{1} \mu_{2}}^{(2,1)^{(0)}}\left(x_{1}, x_{2} ; z\right) V^{\mu_{1}}\left(x_{1}\right) V^{\mu_{2}}\left(x_{2}\right) \\
& +\frac{1}{2} \int d^{4} x_{1} d^{4} x_{2} d^{4} y \Gamma_{\mu_{1} \mu_{2}}^{(2,2,0)}\left(x_{1}, x_{2} ; z, y\right) V^{\mu_{1}}\left(x_{1}\right) V^{\mu_{2}}\left(x_{2}\right) \phi(y) \\
& +\cdots \text {, }
\end{aligned}
$$

where we have used the replacements $V^{\mu} \rightarrow g_{\mathrm{v}}, V^{\mu}$ and $\phi \rightarrow g_{s}, \phi$ in order to establish the connection with the interacting theory. For time-independent external potentials one can, with the standard Fourier transform convention

$$
\begin{aligned}
\Gamma_{\mu_{1} \cdots \mu_{m}}^{(m, n)}\left(x_{1}, \ldots x_{m+n}\right)= & \int \frac{d^{4} k_{1}}{(2 \pi)^{4}} \cdots \frac{d^{4} k_{m+n}}{(2 \pi)^{4}} \exp \left\{-i k_{1} \cdot x_{1}-\cdots-i k_{m+n} \cdot x_{m+n}\right\} \\
& \times(2 \pi)^{4} \delta^{(4)}\left(k_{1}+\cdots+k_{m+n}\right) \Gamma_{\mu_{1} \cdots \mu_{m}}^{(m, n)}\left(k_{1}, \ldots, k_{m+n-1}\right),
\end{aligned}
$$

rewrite Eqs. (3.27), (3.28) as

$$
\begin{aligned}
-i g_{v} j_{v}(z)= & \int d^{3} x \int \frac{d^{3} k}{(2 \pi)^{3}} e^{i k \cdot(x-z)} \Gamma_{v \mu}^{(2,0)(0)}\left(k^{0}=0, \underline{k}\right) V^{\mu}(\underline{x}) \\
& +\cdots
\end{aligned}
$$




$$
\begin{aligned}
& i g_{\mathrm{s}} \rho_{\mathrm{s}}(z)=\Gamma^{\left(0,1 \eta^{(0)}\right.}(\underline{z})+\int d^{3} y \int \frac{d^{3} k}{(2 \pi)^{3}} e^{i k \cdot(\underline{y}-\underline{z})} \Gamma^{\left(0,2 y^{(0)}\right.}\left(k^{0}=0, \underline{k}\right) \phi(\underline{y}) \\
& +\frac{1}{2} \int d^{3} y_{1} d^{3} y_{2} \int \frac{d^{3} k_{1}}{(2 \pi)^{3}} \int \frac{d^{3} k_{2}}{(2 \pi)^{3}} e^{i k_{1} \cdot\left(y_{1}-z\right)} e^{i k_{2} \cdot\left(y_{2}-z\right)} \\
& \times \Gamma^{(0.3)^{\prime 01}}\left(k_{1}^{0}=0, k_{1}, k_{2}^{0}=0, \underline{k}_{2}\right) \phi\left(y_{1}\right) \phi\left(y_{2}\right) \\
& +\frac{1}{6} \int d^{3} y_{1} d^{3} y_{2} d^{3} y_{3} \int \frac{d^{3} k_{1}}{(2 \pi)^{3}} \int \frac{d^{3} k_{2}}{(2 \pi)^{3}} \int \frac{d^{3} k_{3}}{(2 \pi)^{3}} \\
& \times e^{i k_{1} \cdot\left(y_{1}-z\right)} e^{i k_{2} \cdot\left(y_{2}-z\right)} e^{i k_{3} \cdot\left(y_{3}-\underline{z}\right)} \\
& \times \Gamma^{(0,4)^{(0)}}\left(k_{1}^{0}=0, \underline{k}_{1}, k_{2}^{0}=0, \underline{k}_{2}, k_{3}^{0}=0, \underline{k}_{3}\right) \\
& \times \phi\left(\underline{y}_{1}\right) \phi\left(\underline{y}_{2}\right) \phi\left(\underline{y}_{3}\right) \\
& +\cdots \text {, }
\end{aligned}
$$

wherc only the divergent terms of Eqs. (3.27) and (3.28) have been kept. One then realizes that the semiclassical expansions correspond to an expansion of the irreducible functions about points of vanishing momenta,

$$
\begin{aligned}
& -i g_{\mathrm{v}} j_{v}(\underline{z})=\left.\left\{1-\frac{1}{2} \partial_{i}^{z} \partial_{i}^{k} \partial_{j}^{z} \partial_{j}^{k}\right\} \Gamma_{v \neq i}^{(2,0)^{(0)}}(k)\right|_{k=0} V^{\mu}(\underline{z}) \\
& +\cdots \\
& i g_{s} \rho_{\mathrm{s}}(\underline{z})=\Gamma^{(0,1)^{(0)}}(\underline{z})+\left.\left\{1-\frac{1}{2} \partial_{i}^{z} \partial_{i}^{k} \partial_{j}^{z} \partial_{j}^{k}\right\} \Gamma^{(0,2)^{101}}(k)\right|_{k=0} \phi(\underline{z}) \\
& +\frac{1}{2}\left\{1-\partial_{i}^{y_{1}} \partial_{i}^{k_{1}} \partial_{j}^{y_{1}} \partial_{j}^{k_{1}}-\partial_{i}^{y_{1}} \partial_{i}^{k_{1}} \partial_{j}^{y_{2}} \partial_{j}^{k_{2}}\right\} \\
& \times\left.\left.\Gamma^{(0,3)^{(0)}}\left(k_{1}, k_{2}\right)\right|_{k_{1}=k_{2}=0} \phi\left(\underline{y}_{1}\right) \phi\left(\underline{y}_{2}\right)\right|_{y_{1}=y_{2}=z} \\
& +\frac{1}{6}\left\{1-\partial_{i}^{y_{1}} \partial_{i}^{k_{1}} \partial_{j}^{y_{1}} \partial_{j}^{k_{1}}-\partial_{i}^{y_{1}} \partial_{i}^{k_{1}} \partial_{j}^{y_{2}} \partial_{j}^{k_{2}}\right\}
\end{aligned}
$$

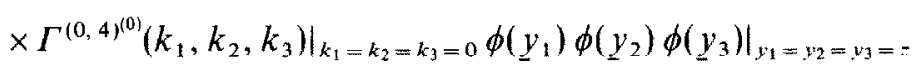

$$
\begin{aligned}
& +\cdots \text {. }
\end{aligned}
$$

In writing Eqs. (3.32), (3.33) the abbreviation

$$
\partial_{i}^{a} \equiv \frac{\partial}{\partial a^{i}}
$$

and the symmetries of the functions $\Gamma^{(0, n)}$ with respect to interchange of their arguments have been used. The expansion of the irreducible functions about the origin in momentum space determines the lowest order gradient contributions to the densities of interest.

The renormalisation of the functions $\Gamma^{(m . n)}$ is most directly discussed in the context of an interacting theory without classical external potentials. For our purposes a renormalisation of the interacting Lagrangian (2.1) at the one-loop level is sufficient. The renormalised $(m, n)$-point functions that are extracted from the one- 
loop renormalisation can then be used for the noninteracting situation, that is in Eqs. (3.32) and (3.33).

The renormalisation procedure itself is carried through in momentum space. We start by representing the exact vector and scalar meson propagators in terms of the corresponding irreducible functions. The former allow a direct physical interpretation whereas we ultimately want to express the normalisation conditions in terms of the latter. For the meson Green's functions

$$
\begin{aligned}
i G_{\mu \nu}^{(2,0)}(x, y) \equiv i D_{\mu \nu}(x-y) & =\left\langle 0\left|T V_{\mu}(x) V_{v}(y)\right| 0\right\rangle \\
i G^{(0,1)}(x) & =\langle 0|\phi(x)| 0\rangle \\
i G^{(0,2)}(x, y) & =\langle 0|T \phi(x) \phi(y)| 0\rangle
\end{aligned}
$$

one directly finds the basic identities

$$
\begin{aligned}
G^{(0,1)}(x) & =\int d^{4} y \Delta(x-y) \Gamma^{(0,1)}(y) \\
G^{(0,2)}(x, y) & =i G^{(0,1)}(x) G^{(0,1)}(y)+\Delta(x-y),
\end{aligned}
$$

where $\Delta(x-y)$ is exactly the connected part of $G^{(0,2)}(x, y)$. Using the tensor structure of the vector meson vacuum polarisation,

$$
-i \Pi_{\mu v}^{\mathrm{v}}(k) \equiv \Gamma_{\mu v}^{(2,0)}(k)=-i\left(k^{2} g_{\mu v}-k_{\mu} k_{v}\right) \Pi_{\mathrm{v}}\left(k^{2}\right),
$$

and the analogous abbreviation

$$
-i \Pi_{\mathrm{s}}(k) \equiv \Gamma^{(0,2)}\left(k^{2}\right),
$$

the Dyson equations for the meson propagators lead to

$$
\begin{aligned}
D_{\mu v}(k) & =\left(g_{\mu v}-\frac{k_{\mu} k_{v}}{k^{2}}\right) \frac{-1}{k^{2}\left(1+\Pi_{\mathrm{v}}\left(k^{2}\right)\right)-m_{v}^{2}+i \varepsilon}+\frac{k_{\mu} k_{v}}{k^{2} m_{\mathrm{v}}^{2}} \\
\Delta(k) & =\frac{1}{k^{2}-m_{\mathrm{s}}^{2}-\Pi_{\mathrm{s}}(k)+i \varepsilon} .
\end{aligned}
$$

As we do not need the corresponding relations for the nucleon propagator and the vector and scalar vertex corrections for our purposes we do not display them here. However, we do need the higher order connected scalar meson Green's functions

$$
\begin{aligned}
G_{\mathrm{c}}^{(0,3)}(k, p)= & \Delta\left(k^{2}\right) \Delta\left(p^{2}\right) \Delta\left((p+k)^{2}\right) \Gamma^{(0,3)}(p, k) \\
G_{\mathrm{c}}^{(0,4)}(k, p, q)= & \Delta\left(k^{2}\right) \Delta\left(p^{2}\right) \Delta\left(q^{2}\right) \Delta\left((p+k+q)^{2}\right) \\
& \times\left\{\Gamma^{(0,4)}(p, k, q)\right. \\
& +\Gamma^{(0,3)}(p,-p-k-q) \Delta\left((k+q)^{2}\right) \Gamma^{(0,3)}(k, q) \\
& +\Gamma^{(0,3)}(k,-p-k-q) \Delta\left((p+q)^{2}\right) \Gamma^{(0,3)}(p, q) \\
& \left.+\Gamma^{(0,3)}(q,-p-k-q) \Delta\left((p+k)^{2}\right) \Gamma^{(0,3)}(p, k)\right\},
\end{aligned}
$$


which characterise higher order scalar meson scattering. It is the renormalisation of the functions (3.34) and (3.38)-(3.41) on the one-loop level by the introduction of suitable counterterms which at the same time yields the renormalisation of the noninteracting problem.

As a first step we need explicit expressions for the irreducible functions indicated above on the one-loop level. All divergent one-loop diagrams of the interacting theory are shown in Fig. 3, where only the graphs (a) and (d-g) are relevant for the following. Fortunately we do not have to evaluate these diagrams for arbitrary external momenta. The only interesting point in momentum space is the renormalisation point where we impose the normalisation conditions. The standard choice for the renormalisation point, which is the most simple and at the same time the point of interest for the semiclassical expansion, is the point of vanishing external momenta. Using dimensional regularisation and expanding around the renormalisation point $k_{\mu}^{i}=0$ one finds for the relevant functions

$$
\begin{aligned}
\Pi_{\mathrm{v}, \mathrm{reg}}^{(0)}\left(k^{2}\right)= & \frac{g_{\mathrm{v}}^{2}}{12 \pi^{2}}\left\{\Gamma\left(2-\frac{D}{2}\right)+2 \ln \left|\frac{m}{M}\right|+\mathcal{O}\left(k^{2}\right)\right\} \\
\Gamma_{\mathrm{reg}}^{(0,1)^{(0)}=}= & i g_{\mathrm{s}} \frac{M^{3}}{4 \pi^{2}}\left\{\Gamma\left(2-\frac{D}{2}\right)+1+2 \ln \left|\frac{m}{M}\right|\right\} \\
\Gamma_{\mathrm{reg}}^{(0,2)^{(0)}}\left(k^{2}\right)= & -i g_{\mathrm{s}}^{2} \frac{M^{2}}{4 \pi^{2}}\left\{3 \Gamma\left(2-\frac{D}{2}\right)+1+6 \ln \left|\frac{m}{M}\right|\right. \\
& \left.+\left[-\frac{1}{2} \Gamma\left(2-\frac{D}{2}\right)+\frac{1}{3}-\ln \left|\frac{m}{M}\right|\right] \frac{k^{2}}{M^{2}}+\mathcal{O}\left(k^{4}\right)\right\} \\
\Gamma_{\mathrm{reg}}^{(0,3)^{(0)}}\left(k_{1}^{\mu}, k_{2}^{\mu}\right)= & 2 i g_{\mathrm{s}}^{3} \frac{M}{4 \pi^{2}}\left\{3 \Gamma\left(2-\frac{D}{2}\right)-2+6 \ln \left|\frac{m}{M}\right|\right. \\
& \left.+\mathcal{O}\left(k_{1}^{2}, k_{2}^{2}, k_{1} \cdot k_{2}\right)\right\} \\
\Gamma_{\mathrm{reg}}^{(0,4)^{(0)}}\left(k_{1}^{\mu}, k_{2}^{\mu}, k_{3}^{\mu}\right)= & -6 i g_{\mathrm{s}}^{4} \frac{1}{4 \pi^{2}}\left\{\Gamma\left(2-\frac{D}{2}\right)-\frac{8}{3}+2 \ln \left|\frac{m}{M}\right|\right. \\
& \left.+\mathcal{O}\left(k_{1}^{2}, k_{2}^{2}, k_{3}^{2}, k_{1} \cdot k_{2}, k_{1} \cdot k_{3}, k_{2} \cdot k_{3}\right)\right\} .
\end{aligned}
$$

Here $m$ is the mass scale introduced by dimensional regularisation and $D$ the dimension of the Minkowski space. We note that there are two topologically distinct diagrams contributing to $\Gamma^{(0,3)}$ and six contributing to $\Gamma^{(0,4)}$ that give identical results for vanishing external momenta. All contributions of higher order in the external momenta which are not displayed explicitly above are finite. This fact is responsible for the success of renormalisation, i.e., it allows the elimination of the divergent terms by introduction of suitable counterterms in the Lagrangian. 


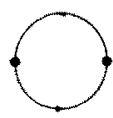

(a)

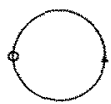

(d)

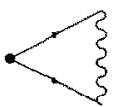

(h)

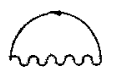

(b)

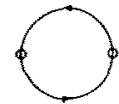

(e)

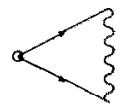

(i)

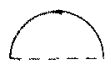

(c)

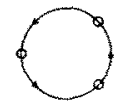

(f)

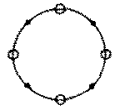

(g)

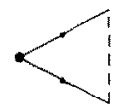

(i)

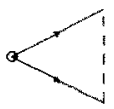

(k)

Fig. 3. Divergent one-loop diagrams of the interacting system: meson propagator, $-\cdots-=$ scalar meson propagator, and $\rightarrow=$ nucleon propagator.

Adding all legitimate counterterms to the Lagrangian,

$$
\begin{aligned}
\mathscr{L}_{\mathrm{R}}= & Z_{\psi} \psi\left(i \gamma^{\mu} \partial_{\mu}-M+\delta M\right) \psi-Z_{g_{v}} g_{\mathrm{v}} \psi \gamma^{\mu} V_{\mu} \psi+Z_{g_{\mathrm{s}}} g_{\mathrm{s}} \psi \phi \psi \\
& +\frac{Z_{\mathrm{s}}}{2}\left(\partial_{\mu} \phi \partial^{\mu} \phi-\left(m_{\mathrm{s}}^{2}-\delta m_{\mathrm{s}}^{2}\right) \phi^{2}\right)+\alpha_{1} \phi+\frac{\alpha_{3}}{3 !} \phi^{3}+\frac{\alpha_{4}}{4 !} \phi^{4} \\
& -\frac{Z_{v}}{4} F^{\mu v} F_{\mu v}+\frac{Z_{v}}{2}\left(m_{v}^{2}-\delta m_{v}^{2}\right) V_{\mu} V^{\mu},
\end{aligned}
$$

leads to additional contributions to all divergent functions. One obtains for the relevant renormalised functions

$$
\begin{aligned}
G_{\mathrm{R}}^{(0,1)}= & \Delta_{\mathrm{R}}\left(k^{2}=0\right)\left\{\Gamma_{\mathrm{reg}}^{(0,1)}+i \alpha_{1}\right\} \\
D_{\mathrm{R}, \mu v}(k)= & Z_{\mathrm{v}}^{-1}\left\{\left(g_{\mu v}-\frac{k_{\mu} k_{v}}{k^{2}}\right) \frac{-1}{k^{2}\left(1+\Pi_{\mathrm{v}, \mathrm{reg}}\left(k^{2}\right)\right)-m_{\mathrm{v}}^{2}+\delta m_{\mathrm{v}}^{2}+i \varepsilon}\right. \\
& \left.+\frac{k_{\mu} k_{\mathrm{v}}}{k^{2}\left(m_{\mathrm{v}}^{2}-\delta m_{\mathrm{v}}^{2}\right)}\right\} \\
\Delta_{\mathrm{R}}(k)= & Z_{\mathrm{s}}^{-1} \frac{1}{k^{2}-m_{\mathrm{s}}^{2}+\delta m_{\mathrm{s}}^{2}-\Pi_{\mathrm{s}, \mathrm{reg}}(k)+i \varepsilon} .
\end{aligned}
$$

These equations are exact to all orders if one uses the bare parameters

$$
\begin{aligned}
g_{0, \mathrm{v}} & =\frac{Z_{g_{\mathrm{v}}}}{Z_{\psi} \sqrt{Z_{\mathrm{v}}}} g_{\mathrm{v}} \\
g_{0, \mathrm{~s}} & =\frac{Z_{g_{\mathrm{s}}}}{Z_{\psi} \sqrt{Z_{\mathrm{s}}}} g_{\mathrm{s}} \\
M_{0} & =M-\delta M \\
m_{0, \mathrm{v}}^{2} & =m_{\mathrm{v}}^{2}-\delta m_{\mathrm{v}}^{2} \\
m_{0, \mathrm{~s}}^{2} & =m_{\mathrm{s}}^{2}-\delta m_{\mathrm{s}}^{2}
\end{aligned}
$$


for the evaluation of $\Gamma_{\mathrm{reg}}^{(0,1)}, \Pi_{\mathrm{v}, \text { reg }}$ and $\Pi_{\mathrm{s} \text {, reg }}$ on the right hand side of Eqs. (3.48)-(3.50) with a subsequent order by order elimination of the bare parameters in favour of the physical ones. To first order, however, one can use the physical parameters directly. The corresponding first order relations for the renormalised higher order scalar meson Green's functions are

$$
\begin{aligned}
G_{\mathrm{c}, \mathrm{R}}^{(0,3)}(k, p)= & \Delta_{\mathrm{R}}\left(k^{2}\right) \Delta_{\mathrm{R}}\left(p^{2}\right) \Delta_{\mathrm{R}}\left((p+k)^{2}\right)\left\{\Gamma_{\mathrm{reg}}^{(0,3)}(p, k)+i \alpha_{3}\right\} \\
G_{\mathrm{c}, \mathrm{R}}^{(0,4)}(k, p, q)= & \Delta_{\mathrm{R}}\left(k^{2}\right) \Delta_{\mathrm{R}}\left(p^{2}\right) \Delta_{\mathrm{R}}\left(q^{2}\right) \Delta_{\mathrm{R}}\left((p+k+q)^{2}\right) \\
& \times\left\{\Gamma_{\mathrm{reg}}^{(0,4)}(p, k, q)+i \alpha_{4}\right. \\
& +\left[\Gamma_{\mathrm{reg}}^{(0,3)}(p,-p-k-q)+i \alpha_{3}\right] \Delta_{\mathrm{R}}\left((k+q)^{2}\right)\left[\Gamma_{\mathrm{reg}}^{(0,3)}(k, q)+i \alpha_{3}\right] \\
& +\left[\Gamma_{\mathrm{reg}}^{(0,3)}(k,-p-k-q)+i \alpha_{3}\right] \Delta_{\mathrm{R}}\left((p+q)^{2}\right)\left[\Gamma_{\mathrm{reg}}^{(0,3)}(p, q)+i \alpha_{3}\right] \\
& \left.+\left[\Gamma_{\mathrm{reg}}^{(0,3)}(q,-p-k-q)+i \alpha_{3}\right] \Delta_{\mathrm{R}}\left((p+k)^{2}\right)\left[\Gamma_{\mathrm{reg}}^{(0,3)}(p, k)+i \alpha_{3}\right]\right\} .
\end{aligned}
$$

Thus to first order the interesting renormalised irreducible functions are given by

$$
\begin{aligned}
\Pi_{\mathrm{v}, \mathrm{R}}\left(k^{2}\right) & =\Pi_{\mathrm{v}, \mathrm{reg}}\left(k^{2}\right)+Z_{\mathrm{v}}-1 \\
\delta m_{\mathrm{v}}^{2} & =\left(Z_{\mathrm{v}}-1\right) m_{\mathrm{v}}^{2} \\
\Gamma_{\mathrm{R}}^{(0,1)} & =\Gamma_{\mathrm{reg}}^{(0,1)}+i \alpha_{1} \\
\Gamma_{\mathrm{R}}^{(0,2)}\left(k^{2}\right) & =-i \Pi_{\mathrm{s}, \mathrm{R}}(k)=\Gamma_{\mathrm{reg}}^{(0,2)}\left(k^{2}\right)+i\left(Z_{\mathrm{s}}-1\right)\left(k^{2}-m_{\mathrm{s}}^{2}\right)+i \delta m_{\mathrm{s}}^{2} \\
\Gamma_{\mathrm{R}}^{(0,3)}\left(k_{1}, k_{2}\right) & =\Gamma_{\mathrm{rcg}}^{(0,3)}\left(k_{1}, k_{2}\right)+i \alpha_{3} \\
\Gamma_{\mathrm{R}}^{(0,4)}\left(k_{1}, k_{2}, k_{3}\right) & =\Gamma_{\mathrm{reg}}^{(0,4)}\left(k_{1}, k_{2}, k_{3}\right)+i \alpha_{4} .
\end{aligned}
$$

When renormalisation is dealt with the major physical question to be addressed is the choice of the renormalisation point and the normalisation condition imposed at this point. In principle, one may use any point in momentum space to normalise a given $(m, n)$-point function, provided the normalisation condition is chosen appropriately. It is only the combination of renormalisation point and normalisation condition that reflects the physical content of the renormalisation scheme. From this perspective it is clear that in a theory like QED the known experimental electron mass and charge as well as the vanishing of the photon mass determine the on-shell normalisation conditions, in other words, given the renormalisation point there is no additional freedom for the choice of the normalisation condition. The situation is different for an effective theory such as QHD, for which the physical content of the parameters entering into the Lagrangian is not fixed a priori. The physical quality of the model depends on the choice of renormalisation point and normalisation conditions. The standard renormalisation point for 
$\Gamma^{(m . n)}\left(k_{1}, \ldots, k_{m+n-1}\right)$ of QHD is $k_{1}=k_{2}=\cdots=k_{m+n-1}=0$ and we follow this standard both for its technical simplicity and for its relation to our gradient expansion problem. However, based on a consistency argument we shall suggest alternative normalisation conditions.

We first note that the condition

$$
\Pi_{v, \mathrm{R}}^{(0)}\left(k^{2}=0\right)=0
$$

together with Eq. (3.54) is nothing but a definition of the vector meson mass parameter $m_{\mathrm{v}}$. Next the relation

$$
\Gamma_{\mathrm{R}}^{\left(0.11^{(0)}\right.}=0
$$

ensures the stability of the vacuum. It is required in order to ensure that there is no scalar density for the case of vanishing scalar meson field. This condition is completely equivalent to using normal ordering in the scalar meson fermion coupling term.

The remaining functions have to be finite at the origin,

$$
\begin{aligned}
& \Gamma_{\mathrm{R}}^{(0,2)^{(0)}}(k=0)=i g_{s}^{2} c_{2}=\Gamma_{\mathrm{reg}}^{(0.2)^{(0)}}(k=0)+i \alpha_{2} \\
& \Gamma_{\mathrm{R}}^{(0,3)^{(0)}}\left(k_{1}=k_{2}=0\right)=i g_{\mathrm{s}}^{3} c_{3}=\Gamma_{\mathrm{reg}}^{(0,3)^{(0)}}\left(k_{1}=k_{2}=0\right)+i \alpha_{3} \\
& \Gamma_{\mathrm{R}}^{(0,4)^{(0)}}\left(k_{1}=k_{2}=k_{3}=0\right)=i g_{\mathrm{s}}^{4} c_{4}=\Gamma_{\mathrm{reg}}^{(0,4)(0)}\left(k_{1}=k_{2}=k_{3}=0\right)+i \alpha_{4} \text {, }
\end{aligned}
$$

where

$$
\alpha_{2}=\delta m_{\mathrm{s}}^{2}-\left(Z_{\mathrm{s}}-1\right) m_{\mathrm{s}}^{2}
$$

Finally the scalar meson propagator has to have a pole with residue 1 at the renormalisation point $k=0$,

$$
\left.\frac{d}{d k^{2}}\left(A_{\mathrm{R}}^{(0,2)}\left(k^{2}\right)\right)^{-1}\right|_{k^{2}=0}=1=Z_{\mathrm{s}}-\left.i \frac{d}{d k^{2}} \Gamma_{\mathrm{reg}}^{(0,2)(0)}\left(k^{2}\right)\right|_{k^{2}=0},
$$

in order to maintain its definition as a propagator. Thus one finds for the renormalisation constants

$$
\begin{aligned}
Z_{\mathrm{v}} & =1-\Pi_{\mathrm{v}, \text { reg }}^{(0)}\left(k^{2}=0\right) \\
\delta m_{\mathrm{v}}^{2} & =-\Pi_{\mathrm{v}, \mathrm{reg}}^{(0)}\left(k^{2}=0\right) m_{\mathrm{v}}^{2} \\
\alpha_{1} & =i \Gamma_{\mathrm{reg}}^{(0,1)} \\
Z_{\mathrm{s}} & =1+\left.i \frac{d}{d k^{2}} \Gamma_{\mathrm{reg}}^{(0,2)^{(0)}}\left(k^{2}\right)\right|_{k^{2}=0}
\end{aligned}
$$




$$
\begin{aligned}
\delta m_{\mathrm{s}}^{2}-\left(Z_{\mathrm{s}}-1\right) m_{\mathrm{s}}^{2} \equiv \alpha_{2} & =g_{\mathrm{s}}^{2} c_{2}+i \Gamma_{\mathrm{reg}}^{(0,2)(0)}\left(k^{2}=0\right) \\
\alpha_{3} & =g_{\mathrm{s}}^{3} c_{3}+i \Gamma_{\mathrm{reg}}^{(0,3)^{(0)}}\left(k_{1}=k_{2}=0\right) \\
\alpha_{4} & =g_{\mathrm{s}}^{4} c_{4}+i \Gamma_{\mathrm{reg}}^{(0,4)^{(0)}}\left(k_{1}=k_{2}=k_{3}=0\right),
\end{aligned}
$$

or explicitly

$$
\begin{aligned}
Z_{\mathrm{v}} & =1-\frac{g_{\mathrm{v}}^{2}}{12 \pi^{2}}\left\{\Gamma\left(2-\frac{D}{2}\right)+2 \ln \left|\frac{m}{M}\right|\right\} \\
\delta m_{\mathrm{v}}^{2} & =-\frac{g_{\mathrm{v}}^{2} m_{\mathrm{v}}^{2}}{12 \pi^{2}}\left\{\Gamma\left(2-\frac{D}{2}\right)+2 \ln \left|\frac{m}{M}\right|\right\} \\
\alpha_{1} & =-g_{\mathrm{s}} \frac{M^{3}}{4 \pi^{2}}\left\{\Gamma\left(2-\frac{D}{2}\right)+1+2 \ln \left|\frac{m}{M}\right|\right\} \\
Z_{\mathrm{s}} & =1-g_{\mathrm{s}}^{2} \frac{1}{4 \pi^{2}}\left\{\frac{1}{2} \Gamma\left(2-\frac{D}{2}\right)-\frac{1}{3}+\ln \left|\frac{m}{M}\right|\right\} \\
\delta m_{\mathrm{s}}^{2}-\left(Z_{\mathrm{s}}-1\right) m_{\mathrm{s}}^{2} \equiv \alpha_{2} & =g_{\mathrm{s}}^{2} \frac{M^{2}}{4 \pi^{2}}\left\{3 \Gamma\left(2-\frac{D}{2}\right)+1+6 \ln \left|\frac{m}{M}\right|\right\}+g_{\mathrm{s}}^{2} c_{2} \\
\alpha_{3} & =-2 g_{\mathrm{s}}^{3} \frac{M}{4 \pi^{2}}\left\{3 \Gamma\left(2-\frac{D}{2}\right)-2+6 \ln \left|\frac{m}{M}\right|\right\}+g_{\mathrm{s}}^{3} c_{3} \\
\alpha_{4} & =6 g_{\mathrm{s}}^{4} \frac{1}{4 \pi^{2}}\left\{\Gamma\left(2-\frac{D}{2}\right)-\frac{8}{3}+2 \ln \left|\frac{m}{M}\right|\right\}+g_{\mathrm{s}}^{4} c_{4} .
\end{aligned}
$$

The standard choice for the constants $c_{2-4}$ is $c_{2}=c_{3}=c_{4}=0$, the argument being that to first order one does not want the counterterms $\phi^{3}$ and $\phi^{4}$ to contribute to scalar meson scattering at the renormalisation point (and defining the scalar meson mass $m_{\mathrm{s}}$ ). However, as already discussed, the renormalisation point is not unique, i.e., these counterterms do show up at all other points in momentum space.

As an alternative to this conventional choice we suggest a derivation of $c_{2-4}$ via the following argument. We first calculate the values of all higher order $\Gamma^{(0, n)^{(0)}}$ (beginning with $\Gamma^{(0,5)^{(0)}}$ ) at vanishing external momenta,

$$
\Gamma^{(0, n)^{(0)}}\left(k_{1}=\cdots=k_{n-1}=0\right)=-\left(i g_{\mathrm{s}}\right)^{n}(n-1) ! \int \frac{d^{4} p}{(2 \pi)^{4}} \operatorname{tr}\left\{\left[i G^{0}(p)\right]^{n}\right\} .
$$

Using

$$
\frac{d^{n-1}}{d M^{n-1}} G^{0}(p)=(n-1) !\left[G^{0}(p)\right]^{n}
$$


one obtains the recurrence formula

$$
\begin{aligned}
\Gamma^{(0, n)^{(0)}\left(k_{1}=\cdots=k_{n-1}=0\right)} & =\left(-g_{\mathrm{s}}\right)^{n-5} \frac{d^{n-5}}{d M^{n-5}} \Gamma_{\mathrm{reg}}^{(0,5)^{(0)}}\left(k_{1}=\cdots=k_{4}=0\right) \\
& =-\frac{12 i}{4 \pi^{2}} g_{\mathrm{s}}^{n} \frac{(n-5) !}{M^{n-4}} \\
& =\left.\frac{-i g_{\mathrm{s}}^{n}}{2 \pi^{2}}(-1)^{n-5} \frac{d^{n-1}}{d z^{n-1}}\left[(M+z)^{3} \ln \left|\frac{M+z}{M}\right|\right]\right|_{z=0},
\end{aligned}
$$

where the form (3.79) is the direct result and Eq. (3.80) is the only possible way to extend Eq. (3.79) to smaller $n$ that does not introduce a new mass scale. These values for all $\Gamma^{(0 . n)^{(0)}}$ with $n \geqslant 5$ at the renormalisation point neither require nor allow renormalisation, as the introduction of $\phi^{n}$ terms with $n \geqslant 5$ in the renormalised Lagrangian is not possible. Thus treating the divergent low order $\Gamma^{(0, n)^{(0)}}$ in consistency with the convergent higher orders suggests that Eq. (3.79) be applied in the form of Eq. (3.80) for $n=1-4$ also. This approach first of all agrees with $\mathrm{Eq}$. (3.60) for the case $n=1$. A contradiction to this normalisation would have questioned our consistency argument seriously. The remaining normalisation conditions are Eqs. (4.69)-(4.71) with

$$
\begin{aligned}
& c_{2}=\frac{M^{2}}{2 \pi^{2}} \\
& c_{3}=-\frac{5 M}{2 \pi^{2}} \\
& c_{4}=\frac{11}{2 \pi^{2}} .
\end{aligned}
$$

The physical consequences of this normalisation will be investigated in a forthcoming publication.

Both the conventional and the new choice for the $c_{2-4}$ lead to finite four-currents and scalar densities. The differcnec betwecn the two renormalisation schemes amounts to a finite renormalisation. With either choice one obtains the renormalised four-current and scalar density on the one-loop level from the renormalised analogs of Eqs. (3.32) and (3.33),

$$
\begin{aligned}
g_{\mathrm{v}} j_{\mathrm{R}}^{v}(z)= & g_{\mathrm{v}} j_{\mathrm{reg}}^{v}(\underline{z})-\left(Z_{\mathrm{v}}-1\right) \partial_{i} F^{i v}(\underline{z}) \\
g_{\mathrm{s}} \rho_{\mathrm{s}, \mathrm{R}}(\underline{z})= & g_{\mathrm{s}} \rho_{\mathrm{s}, \mathrm{reg}}(\underline{z})+\alpha_{1}+\left[\delta m_{\mathrm{s}}^{2}-m_{\mathrm{s}}^{2}\left(Z_{\mathrm{s}}-1\right)\right] \phi(\underline{z}) \\
& +\left(Z_{\mathrm{s}}-1\right)(\Delta \phi(\underline{z}))+\frac{\alpha_{3}}{2}(\phi(\underline{z}))^{2}+\frac{\alpha_{4}}{6}(\phi(z))^{3}
\end{aligned}
$$


which have been written in a form allowing direct application to both the vacuum and any other groundstate. Using these formulae one obtains for the renormalised four-current and scalar density

$$
\begin{aligned}
j_{\mathrm{R}}^{v}(\underline{z})= & \frac{g_{\mathrm{v}}}{6 \pi^{2}} \partial_{i}\left[F^{i v} \ln \left|\frac{M^{*}}{M}\right|\right] \\
\rho_{\mathrm{s}, \mathrm{R}}(\underline{z})= & -\frac{M^{* 3}}{2 \pi^{2}} \ln \left|\frac{M^{*}}{M}\right| \\
& +\left[c_{2}-\frac{M^{2}}{2 \pi^{2}}\right] g_{\mathrm{s}} \phi+\frac{1}{2}\left[c_{3}+\frac{5 M}{2 \pi^{2}}\right]\left(g_{\mathrm{s}} \phi\right)^{2}+\frac{1}{6}\left[c_{4}-\frac{11}{2 \pi^{2}}\right]\left(g_{\mathrm{s}} \phi\right)^{3} \\
& +\frac{g_{\mathrm{v}}^{2}}{24 \pi^{2}} \frac{1}{M^{*}}\left[2\left(\underline{\nabla} V^{0}\right)^{2}-F_{i j} F^{i j}\right]+\frac{g_{\mathrm{s}}^{2}}{8 \pi^{2}} \frac{1}{M^{*}}(\nabla \phi)^{2}-\frac{g_{\mathrm{s}}}{4 \pi^{2}} \ln \left|\frac{M^{*}}{M}\right| \Delta \phi .
\end{aligned}
$$

It is obvious that the choice (3.81)-(3.83) for $c_{2}$ to $c_{4}$ simplifies the scalar density.

Finally the total energy remains to be renormalised. This is most easily achieved by deriving the renormalised Hamiltonian from Eq. (3.47),

$$
\begin{aligned}
\mathscr{H}_{\mathrm{R}}= & Z_{\psi} \psi\left(i \gamma^{0} \partial^{0}\right) \psi+Z_{s}\left(\partial^{0} \phi\right)^{2}-Z_{v}\left(\partial^{0} V_{\rho} \partial^{0} V^{\rho}-\partial_{\rho} V^{0} \partial^{0} V^{\rho}\right) \\
& -\frac{Z_{\mathrm{s}}}{2}\left(\partial_{v} \phi \partial^{v} \phi-\left(m_{\mathrm{s}}^{2}-\delta m_{\mathrm{s}}^{2}\right) \phi^{2}\right)-\alpha_{1} \phi-\frac{\alpha_{3}}{3 !} \phi^{3}-\frac{\alpha_{4}}{4 !} \phi^{4} \\
& +\frac{Z_{v}}{4} F^{\mu v} F_{\mu v}-\frac{Z_{v}}{2}\left(m_{v}^{2}-\delta m_{v}^{2}\right) V^{v} V_{v} .
\end{aligned}
$$

For the interesting case of noninteracting nucleons in classical time-independent external potentials only the one-loop contributions to the $\phi$ - and $V^{y}$-counterterms are relevant,

$$
\begin{aligned}
\delta \mathscr{H}= & -\frac{Z_{\mathrm{s}}-1}{2} \partial_{i} \phi \partial^{i} \phi-\frac{1}{2}\left(\delta m_{\mathrm{s}}^{2}-\left(Z_{\mathrm{s}}-1\right) m_{\mathrm{s}}^{2}\right) \phi^{2}-\alpha_{1} \phi-\frac{\alpha_{3}}{3 !} \phi^{3}-\frac{\alpha_{4}}{4 !} \phi^{4} \\
& +\frac{Z_{\mathrm{v}}-1}{4}\left(2 \partial_{i} V_{0} \partial^{i} V^{0}+F^{i j} F_{i j}\right),
\end{aligned}
$$

where Eq. (3.54) has been used. Thus at the one-loop level the total energy density of this system is given by

$$
\begin{aligned}
\varepsilon_{\mathrm{R}}^{\mathrm{B}}= & \varepsilon_{\mathrm{reg}}^{\mathrm{B}}-\frac{Z_{\mathrm{s}}-1}{2} \partial_{i} \phi \partial^{i} \phi-\frac{1}{2}\left(\delta m_{\mathrm{s}}^{2}-\left(Z_{\mathrm{s}}-1\right) m_{\mathrm{s}}^{2}\right) \phi^{2}-\alpha_{1} \phi-\frac{\alpha_{3}}{3 !} \phi^{3}-\frac{\alpha_{4}}{4 !} \phi^{4} \\
& +\frac{Z_{\mathrm{v}}-1}{4}\left(2 \partial_{i} V_{0} \partial^{i} V^{0}+F^{i j} F_{i j}\right) .
\end{aligned}
$$

These counterterms, accompanied by a subtraction of the free Dirac sea contribu- 
tion, cancel the divergencies in Eq. (3.23) exactly, so that the final result for the vacuum energy density is

$$
\begin{aligned}
\varepsilon_{\mathrm{R}}^{\mathrm{B}}= & \frac{M^{* 4}}{32 \pi^{2}}\left\{1-4 \ln \left|\frac{M^{*}}{M}\right|\right\}-\frac{M^{4}}{32 \pi^{2}} \\
& -\frac{1}{2}\left[c_{2}-\frac{M^{2}}{2 \pi^{2}}\right]\left(g_{\mathrm{s}} \phi\right)^{2}-\frac{1}{6}\left[c_{3}+\frac{5 M}{2 \pi^{2}}\right]\left(g_{\mathrm{s}} \phi\right)^{3}-\frac{1}{24}\left[c_{4}-\frac{11}{2 \pi^{2}}\right]\left(g_{\mathrm{s}} \phi\right)^{4} \\
& +\frac{g_{\mathrm{v}}^{2}}{24 \pi^{2}} \ln \left|\frac{M^{*}}{M}\right|\left[2\left(\nabla V^{0}\right)^{2}-F_{i j} F^{i j}\right]-\frac{g_{\mathrm{s}}^{2}}{8 \pi^{2}} \ln \left|\frac{M^{*}}{M}\right|(\nabla \phi)^{2}
\end{aligned}
$$

As for the scalar density, the choice (3.81)-(3.83) for $c_{2}$ to $c_{4}$ simplifies the energy density considerably. The merits of this normalisation scheme compared to the usual choice $c_{2}=c_{3}=c_{4}=0$ remain to be investigated in more detail.

The second order vacuum contributions given in Eqs. (3.86), (3.87), (3.91) are independent of the specific normalisation. They are identical with the results obtained by Perry [10] and Wasson [11] for the case of stationary potentials.

\subsection{Inversion}

As the final step of the program outlined we have to eliminate the semiclassical quantities $V_{\mu}$ and $M^{*}$ in favour of the four-current $j_{\mu}$ and the scalar density $\rho_{\mathrm{s}}$. For this purpose one starts with the (renormalised) zero order results for the densities given in Eqs. (3.21), (3.22), respectively

$$
\begin{aligned}
\rho^{[0]}= & f\left(k_{\mathrm{F}}\right)=\frac{1}{3 \pi^{2}} k_{\mathrm{F}}^{3} \\
\rho_{\mathrm{s}}^{[0]}= & g\left(k_{\mathrm{F}}, M^{*}\right)=\frac{1}{2 \pi^{2}} M^{*}\left(k_{\mathrm{F}} E_{\mathrm{F}}^{*}-M^{* 2} \ln \frac{k_{\mathrm{F}}+E_{\mathrm{F}}^{*}}{M^{*}}\right) \\
& -\frac{M^{* 3}}{2 \pi^{2}} \ln \left|\frac{M^{*}}{M}\right|+\left(c_{2}-\frac{M^{2}}{2 \pi^{2}}\right)\left(M-M^{*}\right) \\
& +\frac{1}{2}\left(c_{3}+\frac{5 M}{2 \pi^{2}}\right)\left(M-M^{*}\right)^{2}+\frac{1}{6}\left(c_{4}-\frac{11}{2 \pi^{2}}\right)\left(M-M^{*}\right)^{3} .
\end{aligned}
$$

There are no zero order contributions to the spatial part of the four-current. The second order contributions can be treated in the same manner as in the case of QED [14], as indicated below.

One then defines two auxiliary variables $\tilde{k}_{\mathrm{F}}$ and $\tilde{M}$, so that the exact densities are given by the functional forms (3.92) with the replacements

$$
\begin{aligned}
& \rho \equiv f\left(\tilde{k}_{\mathrm{F}}\right) \\
& \rho_{\mathrm{s}} \equiv g\left(\tilde{k}_{\mathrm{F}}, \tilde{M}\right) .
\end{aligned}
$$

The basic requirement, that this transformation of variables be invertible, is trivially satisfied for the density $\rho$ and can be demonstrated explicitly for the scalar density 
$\rho_{\mathrm{s}}$ if only valence nucleon contributions are taken into account. It is not a priori satisfied for the full, renormalised form of $\rho_{\mathrm{s}}$ in either normalisation scheme. One expects, however, that the solution of the variational problem (see Section 4) leads to a range of values of the variables $\widetilde{k}_{\mathrm{F}}$ and $\tilde{M}$ such that unique invertibility is guaranteed. If this is not the case, alternative inversion schemes have to be considered.

Keeping in mind that this caveat has to be ascertained a posteriori, one proceeds in the standard fashion. Introducing the expansions

$$
\begin{aligned}
k_{\mathrm{F}} & =\tilde{k}_{\mathrm{F}}-\tilde{k}_{\mathrm{F}}^{[2]} \\
M^{*} & =\tilde{M}-\tilde{M}^{[2]}
\end{aligned}
$$

into the initial semiclassical expansion of the densities

$$
\begin{aligned}
\rho & =\rho^{[0]}\left(k_{\mathrm{F}}\right)+\rho^{[2]}\left(k_{\mathrm{F}}, M^{*}, F^{2}\right) \\
\rho_{\mathrm{s}} & =\rho_{\mathrm{s}}^{[0]}\left(k_{\mathrm{F}}, M^{*}\right)+\rho_{\mathrm{s}}^{[2]}\left(k_{\mathrm{F}}, M^{*}, F^{2}\right)
\end{aligned}
$$

yields to second order the relations

$$
\begin{gathered}
k_{\mathrm{F}}=\tilde{k}_{\mathrm{F}}-\left(\frac{\partial f\left(\tilde{k}_{\mathrm{F}}\right)}{\partial \tilde{k}_{\mathrm{F}}}\right)^{-1} \rho^{[2]}\left(\tilde{k}_{\mathrm{F}}, \tilde{M}, F^{2}\right) \\
M^{*}=\tilde{M}-\left(\frac{\partial g\left(\tilde{k}_{\mathrm{F}}, \tilde{M}\right)}{\partial \tilde{M}}\right)^{-1} \alpha^{[2]}\left(\tilde{k}_{\mathrm{F}}, \tilde{M}, F^{2}\right),
\end{gathered}
$$

with

$$
\alpha^{[2]}\left(\tilde{k}_{\mathrm{F}}, \tilde{M}, F^{2}\right) \equiv \rho_{\mathrm{s}}^{[2]}\left(\tilde{k}_{\mathrm{F}}, \tilde{M}, F^{2}\right)-\left(\frac{\partial f\left(\tilde{k}_{\mathrm{F}}\right)}{\partial \tilde{k}_{\mathrm{F}}}\right)^{-1}\left(\frac{\partial g\left(\tilde{k}_{\mathrm{F}}, \tilde{M}\right)}{\partial \tilde{k}_{\mathrm{F}}}\right) \rho^{[2]}\left(\tilde{k}_{\mathrm{F}}, \tilde{M}, F^{2}\right) .
$$

The relations (3.96) allow the elimination of $k_{\mathrm{F}}$ and $M^{*}$ from the semiclassical gradient expansion of the energy density (3.23).

For the elimination of the field tensor in terms of the spatial current we note that the vector current after renormalisation is given by (see Eqs. (3.21), (3.86))

$$
j^{k}(\underline{x})=\frac{1}{6 \pi^{2}} \partial_{i}\left[F^{i k}(\underline{x}) \ln \frac{k_{\mathrm{F}}+E_{\mathrm{F}}^{*}}{M}\right] .
$$

As the current is of second order in $\hbar$ one can directly replace $k_{\mathrm{F}}$ and $M^{*}$ by $\tilde{k}_{\mathrm{F}}$ and $\tilde{M}$. With the definition

$$
\widetilde{E}_{\mathrm{F}} \equiv \sqrt{\tilde{k}_{\mathrm{F}}^{2}+\widetilde{M}^{2}}
$$

Eq. (3.98) is resolved by the nonlocal functional

$$
F^{i k}(\underline{x})=\frac{3 \pi}{2}\left(\ln \frac{\tilde{k}_{\mathrm{F}}+\tilde{E}_{\mathrm{F}}}{M}\right)^{-1} \int d^{3} y\left\{\partial_{x}^{i} \frac{1}{|\underline{x}-\underline{y}|} j^{k}(\underline{y})-\partial_{x}^{k} \frac{1}{|\underline{x}-\underline{y}|} j^{i}(\underline{y})\right\} .
$$


For the elimination of the semiclassical quantities from the baryonic energy functional, Eq. (3.23), one rewrites Eq. (3.23) as

$$
\varepsilon^{\mathrm{B}}=\rho V_{0}+\varepsilon^{[0]}\left(k_{\mathrm{F}}, M^{*}\right)+\varepsilon^{[2]}\left(k_{\mathrm{F}}, M^{*}, F^{2}\right),
$$

where $\varepsilon^{[0]}$ and $\varepsilon^{[2]}$ are the zeroth and second order contributions to $\varepsilon^{\mathrm{B}}-\rho V_{0}$. Using the relations (3.96) one obtains

$$
\begin{aligned}
\varepsilon^{\mathrm{B}}= & \rho V_{0}+\varepsilon^{[0]}\left(\tilde{k}_{\mathrm{F}}, \tilde{M}\right)+\varepsilon^{[2]}\left(\tilde{k}_{\mathrm{F}}, \tilde{M}, F^{2}\right) \\
& -\frac{\partial \varepsilon^{[0]}\left(\tilde{k}_{\mathrm{F}}, \tilde{M}\right)}{\partial \tilde{k}_{\mathrm{F}}}\left(\frac{\partial f\left(\tilde{k}_{\mathrm{F}}\right)}{\partial \tilde{k}_{\mathrm{F}}}\right)^{-1} \rho^{[2]}\left(\tilde{k}_{\mathrm{F}}, \tilde{M}, F^{2}\right) \\
& -\frac{\partial \varepsilon^{[0]}\left(\tilde{k}_{\mathrm{F}}, \tilde{M}\right)}{\partial \tilde{M}}\left(\frac{\partial g\left(\tilde{k}_{\mathrm{F}}, \tilde{M}\right)}{\partial \tilde{M}}\right)^{-1} \alpha^{[2]}\left(\tilde{k}_{\mathrm{F}}, \tilde{M}, F^{2}\right),
\end{aligned}
$$

where finally $F^{2}$ is replaced via Eq. (3.99). Now the potential energies are subtracted from the baryonic energy in order to derive the kinetic energy density,

$$
t_{\mathrm{s}}=\varepsilon^{\mathrm{B}}-j^{\mu} V_{\mu}+\rho_{\mathrm{s}} \phi
$$

Due to the relation

$$
\frac{\partial \varepsilon^{[0]}\left(\tilde{k}_{\mathrm{F}}, \tilde{M}\right)}{\partial \tilde{M}}=g\left(\tilde{k}_{\mathrm{F}}, \tilde{M}\right)=\rho_{\mathrm{s}}
$$

terms $\sim \alpha^{[2]}$ vanish and one finally ends up with

$$
\begin{aligned}
t_{\mathrm{s}}\left(\tilde{k}_{\mathrm{F}}, \tilde{M}, j^{k}\right) & =t_{\mathrm{s}}^{[0]}\left(\tilde{k}_{\mathrm{F}}, \tilde{M}\right)+t_{\mathrm{s}}^{[2]}\left(\tilde{k}_{\mathrm{F}}, \tilde{M}, j\right) \\
t_{\mathrm{s}}^{[0]}\left(\tilde{k}_{\mathrm{F}}, \tilde{M}\right) & =\varepsilon^{[0]}\left(\tilde{k}_{\mathrm{F}}, \tilde{M}\right)+g\left(\tilde{k}_{\mathrm{F}}, \tilde{M}\right)(M-\tilde{M}) \\
t_{\mathrm{s}}^{[2]}\left(\tilde{k}_{\mathrm{F}}, \tilde{M}, j^{k}\right) & =\varepsilon^{[2]}\left(\tilde{k}_{\mathrm{F}}, \tilde{M}, j^{k}\right)-\tilde{E}_{\mathrm{F}} \rho^{[2]}\left(\tilde{k}_{\mathrm{F}}, \tilde{M}, j^{k}\right)-j^{i} V_{i} .
\end{aligned}
$$

Vacuum and valence nucleon contributions are explicitly given by

$$
\begin{aligned}
t_{\mathrm{s}, \mathrm{R}}^{[0]}\left(\tilde{k}_{\mathrm{F}}, \tilde{M}\right)= & \frac{1}{8 \pi^{2}}\left(3-4 \frac{M}{\tilde{M}}\right) \tilde{M}^{4} \ln \left|\frac{\tilde{M}}{M}\right| \\
& +\frac{1}{32 \pi^{2}}\left(\tilde{M}^{4}-M^{4}\right)+\frac{1}{2}\left(c_{2}-\frac{M^{2}}{2 \pi^{2}}\right)(M-\tilde{M})^{2} \\
& +\frac{1}{3}\left(c_{3}+\frac{5 M}{2 \pi^{2}}\right)(M-\tilde{M})^{3}+\frac{1}{8}\left(c_{4}-\frac{11}{2 \pi^{2}}\right)(M-\tilde{M})^{4} \\
t_{\mathrm{s} . \bar{D}}^{[0]}\left(\tilde{k}_{\mathrm{F}}, \tilde{M}\right)= & \frac{1}{8 \pi^{2}}\left\{\left(4 \frac{M}{\tilde{M}}-3\right) \tilde{k}_{\mathrm{F}} \tilde{E}_{\mathrm{F}}^{3}+\left(5-4 \frac{M}{\tilde{M}}\right) \tilde{k}_{\mathrm{F}}^{3} \tilde{E}_{\mathrm{F}}\right. \\
& \left.+\left(3-4 \frac{M}{\tilde{M}}\right) \tilde{M}^{4} \ln \frac{\tilde{k}_{\mathrm{F}}+\tilde{E}_{\mathrm{F}}}{\tilde{M}}\right\}
\end{aligned}
$$




$$
\begin{aligned}
& t_{\mathrm{s}, \mathrm{R}}^{[2]}\left(\tilde{k}_{\mathrm{F}}, \tilde{M}, j^{k}\right)=\frac{1}{12 \pi^{2}} \frac{\tilde{k}_{\mathrm{F}}^{2}}{\widetilde{E}_{\mathrm{F}}^{2}} \ln \left|\frac{\tilde{M}}{M}\right|\left(\nabla \tilde{k}_{\mathrm{F}}\right)^{2}-\frac{1}{24 \pi^{2}}\left(1+2 \frac{\tilde{k}_{\mathrm{F}}^{2}}{\widetilde{E}_{\mathrm{F}}^{2}}\right) \ln \left|\frac{\tilde{M}}{M}\right|(\underline{\tilde{M}})^{2} \\
& +\frac{1}{6 \pi^{2}} \frac{\tilde{k}_{\mathrm{F}} \tilde{M}}{\tilde{E}_{\mathrm{F}}^{2}} \ln \left|\frac{\tilde{M}}{M}\right|\left(\nabla \tilde{k}_{\mathrm{F}}\right)(\nabla \tilde{M})+\frac{1}{24 \pi^{2}} \ln \left|\frac{\tilde{M}}{M}\right| F_{i j} F^{i j} \\
& t_{\mathrm{s}, \mathrm{D}}^{[2]}\left(\tilde{k}_{\mathrm{F}}, \tilde{M}, j^{k}\right)=\frac{1}{24 \pi^{2}}\left(\frac{\tilde{k}_{\mathrm{F}}}{\widetilde{E}_{\mathrm{F}}}+2 \frac{\widetilde{k}_{\mathrm{F}}^{2}}{\widetilde{E}_{\mathrm{F}}^{2}} \ln \frac{\widetilde{k}_{\mathrm{F}}+\tilde{E}_{\mathrm{F}}}{\tilde{M}}\right)\left(\nabla \tilde{k}_{\mathrm{F}}\right)^{2} \\
& +\frac{1}{24 \pi^{2}}\left(\frac{\tilde{k}_{\mathrm{F}}}{\widetilde{E}_{\mathrm{F}}}-\left(1+2 \frac{\tilde{k}_{\mathrm{F}}^{2}}{\widetilde{E}_{\mathrm{F}}^{2}}\right) \ln \frac{k_{\mathrm{F}}+\tilde{E}_{\mathrm{F}}}{\tilde{M}}\right)(\nabla \tilde{M})^{2} \\
& +\frac{1}{6 \pi^{2}} \frac{\tilde{k}_{\mathrm{F}} \tilde{M}}{\widetilde{E}_{\mathrm{F}}^{2}} \ln \frac{\tilde{k}_{\mathrm{F}}+\tilde{E}_{\mathrm{F}}}{\tilde{M}}\left(\nabla \tilde{k}_{\mathrm{F}}\right)(\nabla \tilde{M})+\frac{1}{24 \pi^{2}} \ln \frac{\tilde{k}_{\mathrm{F}}+\tilde{E}_{\mathrm{F}}}{\tilde{M}} F_{i j} F^{i j} .
\end{aligned}
$$

We note that inclusion of the renormalised vacuum contributions leads to a replacement of $\tilde{M}$ by $M$ in the $\ln \left(\left(\widetilde{K}_{\mathrm{F}}+\widetilde{E}_{\mathrm{F}}\right) / \widetilde{M}\right)$ terms occuring in the valence nucleon part. This kinetic energy functional complements the functional given by Centelles et al. [7] in the sense that both semiclassical quantities $k_{\mathrm{F}}$ and $M^{*}$ have been eliminated in favour of $\tilde{k}_{\mathrm{F}}$ and $\tilde{M}$, which are directly related to the densities $\rho$ and $\rho_{\mathrm{s}}$. Furthermore the four-current contributions are taken into account fully to second order. The kinetic energy functional reduces to the QED result of [14] for $\tilde{M}=M$. The nonrelativistic limit of the functional (3.104)-(3.107) represents an extension of the semi-inverted functional given by Grammaticos and Voros [28]. In contrast to the result of these authors, however, it also contains all nonrelativistic contributions due to the spin part of the current density.

For the discussion of variational equations in the following section we finally note the functional derivatives

$$
\begin{aligned}
\frac{\delta}{\delta \tilde{k}_{\mathrm{F}}} T_{\mathrm{s}}^{[0]}\left(\tilde{k}_{\mathrm{F}}, \tilde{M}\right) & =\frac{\partial f\left(\tilde{k}_{\mathrm{F}}\right)}{\partial \tilde{k}_{\mathrm{F}}} \widetilde{E}_{\mathrm{F}}+\frac{\partial g\left(\tilde{k}_{\mathrm{F}}, \tilde{M}\right)}{\partial \tilde{k}_{\mathrm{F}}}(M-\tilde{M}) \\
\frac{\delta}{\delta \tilde{M}} T_{\mathrm{s}}^{[0]}\left(\tilde{k}_{\mathrm{F}}, \tilde{M}\right) & =\frac{\partial g\left(\tilde{k}_{\mathrm{F}}, \tilde{M}\right)}{\partial \tilde{M}}(M-\tilde{M}) \\
\frac{\delta}{\delta \tilde{k}_{\mathrm{F}}} T_{\mathrm{s}}^{[2]}\left(\tilde{k}_{\mathrm{F}}, \tilde{M}, j^{k}\right) & =-\frac{\tilde{k}_{\mathrm{F}}}{\tilde{E}_{\mathrm{F}}} \rho^{[2]}\left(\tilde{k}_{\mathrm{F}}, \tilde{M}, j^{k}\right) \\
\frac{\delta}{\delta \tilde{M}} T_{\mathrm{s}}^{[2]}\left(\tilde{k}_{\mathrm{F}}, \tilde{M}, j^{k}\right) & =\alpha^{[2]}\left(\tilde{k}_{\mathrm{F}}, \tilde{M}, j^{k}\right) \\
\frac{\delta}{\delta j^{l}} T_{\mathrm{s}}^{[2]}\left(\tilde{k}_{\mathrm{F}}, \tilde{M}, j^{k}\right) & =-\frac{1}{4 \pi} \int d^{3} y \frac{1}{|\underline{x}-\underline{y}|} \partial_{y}^{i} F_{i l}(y) .
\end{aligned}
$$




\section{ETF VARIATIONAL EQUATIONS}

The original variational equations

$$
\begin{aligned}
& 0=\frac{\delta}{\delta \rho(\underline{y})}\left\{E\left[\rho, \rho_{\mathrm{s}}, j^{k}\right]-\mu \int d^{3} x \rho(\underline{x})\right\} \\
& 0=\frac{\delta}{\delta \rho_{\mathrm{s}}(\underline{y})} E\left[\rho, \rho_{\mathrm{s}}, j^{k}\right] \\
& 0=\frac{\delta}{\delta j^{l}(\underline{y})} E\left[\rho, \rho_{\mathrm{s}}, j^{k}\right]
\end{aligned}
$$

are readily transformed to the new set of variables $\tilde{k}_{\mathrm{F}}$ and $\tilde{M}$

$$
\begin{array}{r}
\frac{\delta}{\delta \tilde{k}_{\mathrm{F}}(y)}\left\{E\left[\rho\left[\tilde{k}_{\mathrm{F}}\right], \rho_{\mathrm{s}}\left[\tilde{k}_{\mathrm{F}}, \tilde{M}\right], j^{k}\right]-\mu \int d^{3} x \rho\left[\tilde{k}_{\mathrm{F}}\right](\underline{x})\right\} \\
=\left\{\frac{\partial \rho\left(\tilde{k}_{\mathrm{F}}\right)}{\partial \tilde{k}_{\mathrm{F}}} \frac{\delta}{\delta \rho(\underline{y})}+\frac{\partial \rho_{\mathrm{s}}\left(\tilde{k}_{\mathrm{F}}, \tilde{M}\right)}{\delta \tilde{k}_{\mathrm{F}}} \frac{\delta}{\delta \rho_{\mathrm{s}}(\underline{y})}\right\}\left[E\left[\rho, \rho_{\mathrm{s}}, j^{k}\right]-\mu \int d^{3} x \rho(\underline{x})\right]=0 \\
\frac{\delta}{\delta \tilde{M}(\underline{y})} E\left[\rho\left[\tilde{k}_{\mathrm{F}}\right], \rho_{\mathrm{s}}\left[\tilde{k}_{\mathrm{F}}, \tilde{M}\right], j^{k}\right]=\frac{\partial \rho_{\mathrm{s}}\left(\tilde{k}_{\mathrm{F}}, \tilde{M}\right)}{\partial \tilde{M}} \frac{\delta}{\delta \rho_{\mathrm{s}}(\underline{y})} E\left[\rho, \rho_{\mathrm{s}}, j^{k}\right]=0 \\
\frac{\delta}{\delta j^{\prime}(\underline{y})} E\left[\rho\left[\tilde{k}_{\mathrm{F}}\right], \rho_{\mathrm{s}}\left[\tilde{k}_{\mathrm{F}}, \tilde{M}\right], j^{k}\right]=0 .
\end{array}
$$

They are equivalent to the initial set, provided the transformation from $\rho$ and $\rho_{\mathrm{s}}$ to $\tilde{k}_{\mathrm{F}}$ and $\tilde{M}$ is unique and the partial derivatives $\partial \rho / \partial \tilde{k}_{\mathrm{F}}, \partial \rho_{\mathrm{s}} / \partial \tilde{k}_{\mathrm{F}}$, and $\partial \rho_{\mathrm{s}} / \partial \tilde{M}$ do not vanish. All relevant boundary conditions for $\rho$ and $\rho_{\mathrm{s}}$ have to be reformulated in terms of $\tilde{k}_{\mathrm{F}}$ and $\tilde{M}$.

As an explicit illustration we present the variational equations for the extended Thomas-Fermi (ETF) model. In this approximation the exchange-correlation contribution $E_{x c}$ in Eq. (2.33) is neglected completely and only the Hartree term is kept. For a stationary system the Hartree contribution can be simplified by integration over the time variable,

$$
\begin{aligned}
E_{\mathrm{H}}\left[\tilde{k}_{\mathrm{F}}, \tilde{M}, j^{k}\right]= & +\frac{1}{2} \frac{g_{\mathrm{v}}^{2}}{4 \pi} \int d^{3} x d^{3} y \rho\left[\tilde{k}_{\mathrm{F}}\right](\underline{x}) \frac{e^{-m_{\mathrm{v}}|\underline{x}-\underline{y}|}}{|\underline{x}-\underline{y}|} \rho\left[\tilde{k}_{\mathrm{F}}\right](\underline{y}) \\
& -\frac{1}{2} \frac{g_{\mathrm{s}}^{2}}{4 \pi} \int d^{3} x d^{3} y \rho_{\mathrm{s}}\left[\tilde{k}_{\mathrm{F}}, \tilde{M}\right](\underline{x}) \frac{e^{-m_{\mathrm{s}}|x-\underline{y}|}}{|\underline{x}-\underline{y}|} \rho_{\mathrm{s}}\left[\tilde{k}_{\mathrm{F}}, \tilde{M}\right](\underline{y}) \\
& -\frac{1}{2} \frac{g_{\mathrm{v}}^{2}}{4 \pi} \int d^{3} x d^{3} y \underline{j}(\underline{x}) \frac{e^{-m_{\mathrm{v}}|\underline{\underline{x}}-\underline{y}|}}{|\underline{x}-\underline{y}|} \underline{j}(\underline{y}),
\end{aligned}
$$

leading to a replacement of the meson propagators by static Yukawa potentials.

The simplest ETF approximation is defined by the energy functional

$$
E_{\mathrm{ETF}}\left[\tilde{k}_{\mathrm{F}}, \tilde{M}, j^{k}\right]=T_{s}\left[\tilde{k}_{F}, \tilde{M}, j^{k}\right]+E_{H}\left[\tilde{k}_{\mathrm{F}}, \tilde{M}, j^{k}\right] .
$$


Using the functional derivatives (3.108) of the kinetic energy contribution one finds the variational equations

$$
\begin{aligned}
0= & \frac{\partial \rho_{\mathrm{s}}\left(\tilde{k}_{\mathrm{F}}, \tilde{M}\right)}{\partial \tilde{k}_{\mathrm{F}}}\left(M-\tilde{M}-g_{\mathrm{s}} \chi\right)+\frac{\partial \rho\left(\tilde{k}_{\mathrm{F}}\right)}{\partial \tilde{k}_{\mathrm{F}}}\left(\tilde{E}_{\mathrm{F}}+g_{\mathrm{v}} U_{0}-\mu\right) \\
& -\frac{\tilde{k}_{\mathrm{F}}}{\tilde{E}_{\mathrm{F}} \rho^{[2]}\left(\tilde{k}_{\mathrm{F}}, \tilde{M}, j^{k}\right)} \\
0= & \frac{\partial \rho_{\mathrm{s}}\left(\tilde{k}_{\mathrm{F}}, \tilde{M}\right)}{\partial \tilde{M}}\left(M-\tilde{M}-g_{\mathrm{s}} \chi\right)+\alpha^{[2]}\left(\tilde{k}_{\mathrm{F}}, \tilde{M}, j^{k}\right) \\
0= & g_{\mathrm{v}} U^{k}(\underline{x})-\frac{3}{8} \partial_{i}^{x} \int d^{3} z \frac{1}{|\underline{x}-\underline{z}|}\left(\ln \frac{\tilde{k}_{\mathrm{F}}(\underline{z})+\tilde{E}_{\mathrm{F}}(\underline{z})}{M}\right)^{-1} \\
& \times \int d^{3} y\left\{\partial_{z}^{i} \frac{j^{k}(\underline{y})}{|\underline{z}-\underline{y}|}-\partial_{z}^{k} \frac{j^{i}(\underline{y})}{|\underline{z}-\underline{y}|}\right\} .
\end{aligned}
$$

As usual for such variational equations the potentials

$$
\begin{aligned}
g_{\mathrm{s}} \chi(\underline{x}) & =\frac{g_{\mathrm{s}}^{2}}{4 \pi} \int d^{3} y \frac{e^{-m_{\mathrm{s}}|\underline{x}-\underline{y}|}}{|\underline{x}-\underline{y}|} \rho_{\mathrm{s}}(\underline{y}) \\
g_{\mathrm{v}} U^{\mu}(\underline{x}) & =\frac{g_{\mathrm{v}}^{2}}{4 \pi} \int d^{3} y \frac{e^{-m_{\mathrm{v}} \mid \underline{x}-\underline{\underline{y} \mid}}}{|\underline{x}-\underline{y}|} j^{\mu}(\underline{y})
\end{aligned}
$$

which are solutions of the Helmholtz equations

$$
\begin{aligned}
\left(\Delta-m_{\mathrm{s}}^{2}\right) \chi(\underline{x}) & =-g_{\mathrm{s}} \rho_{\mathrm{s}}(\underline{x}) \\
\left(\Delta-m_{\mathrm{v}}^{2}\right) U^{\mu}(\underline{x}) & =-g_{\mathrm{v}} j^{\mu}(\underline{x})
\end{aligned}
$$

have been introduced.

In order to reduce Eq. (4.11) fully to differential form we define

$$
T_{j}(\underline{x}) \equiv \frac{3}{8} \int d^{3} z \frac{1}{|\underline{x}-\underline{z}|}\left(\ln \frac{\tilde{K}_{\mathrm{F}}(\underline{z})+\tilde{E}_{\mathrm{F}}(\underline{z})}{M}\right)^{-1} \varepsilon_{j m n} \partial_{z}^{m} \omega^{n}(\underline{z})
$$

with

$$
\omega^{n}(z)=\int d^{3} y \frac{j^{n}(y)}{|\underline{z}-\underline{y}|}
$$

so that Eq. (4.11) can be rewritten as

$$
g_{\mathrm{v}} U^{k}(\underline{x})=\varepsilon_{k f i} \partial_{i}^{x} T_{j}(\underline{x}) .
$$

Equation (4.15) has to be solved in conjunction with the differential equations

$$
\Delta T_{j}(\underline{x})=-\frac{3 \pi}{2}\left(\ln \frac{\tilde{k}_{\mathrm{F}}(\underline{x})+\tilde{E}_{\mathrm{F}}(\underline{x})}{M}\right)^{-1} \varepsilon_{j m n} \partial_{x}^{m} \omega^{n}(\underline{x})
$$


and

$$
\Delta \omega^{n}(\underline{x})=-4 \pi j^{n}(\underline{x})
$$

The second order contributions in Eqs. (4.9) and (4.10) are given explicitly by

$$
\begin{aligned}
& \rho^{[2]}\left(\tilde{k}_{\mathrm{F}}, \tilde{M}, j^{k}\right)=\rho_{\mathrm{R}}^{[2]}\left(\tilde{k}_{\mathrm{F}}, \tilde{M}\right)+\rho_{\mathrm{D}}^{[2]}\left(\tilde{k}_{\mathrm{F}}, \tilde{M}, j^{k}\right) \\
& \rho \hbar_{\mathrm{k}}^{2]}\left(\tilde{k}_{\mathrm{F}}, \tilde{M}\right)=\frac{1}{6 \pi^{2}} \frac{\tilde{k}_{\mathrm{F}}}{\tilde{E}_{\mathrm{F}}} \ln \left|\frac{\tilde{M}}{M}\right| \Delta \tilde{k}_{\mathrm{F}}+\frac{1}{6 \pi^{2}} \frac{\tilde{M}^{2}}{\tilde{E}_{\mathrm{F}}^{3}} \ln \left|\frac{\tilde{M}}{M}\right|\left(\underline{\nabla} \tilde{k}_{\mathrm{F}}\right)^{2} \\
& +\frac{1}{6 \pi^{2}} \frac{\tilde{M}}{\tilde{E}_{\mathrm{F}}} \ln \left|\begin{array}{c}
\tilde{M} \\
M
\end{array}\right| \Delta \tilde{M}+\frac{1}{6 \pi^{2}} \frac{1}{\tilde{E}_{\mathrm{F}}}\left(1+\frac{\tilde{k}_{\mathrm{F}}^{2}}{\tilde{E}_{\mathrm{F}}^{2}} \ln \left|\frac{\tilde{M}}{M}\right|\right)(\underline{\nabla})^{2} \\
& +\frac{1}{6 \pi^{2}} \frac{\tilde{k}_{\mathrm{F}}}{\tilde{M} \tilde{E}_{\mathrm{F}}}\left(1-2 \frac{\tilde{M}^{2}}{\tilde{E}_{\mathrm{F}}^{2}} \ln \left|\frac{\tilde{M}}{M}\right|\right)\left(\nabla \tilde{k}_{\mathrm{F}}\right) \cdot(\underline{\nabla} \tilde{M}) \\
& \rho_{\mathrm{D}}^{[2]}\left(\tilde{k}_{\mathrm{F}}, \tilde{M}, j^{k}\right)=\frac{1}{12 \pi^{2}}\left(1+2 \frac{\tilde{k}_{\mathrm{F}}}{\tilde{E}_{\mathrm{F}}} \ln \frac{\tilde{k}_{\mathrm{F}}+\tilde{E}_{\mathrm{F}}}{\tilde{M}}\right) \Delta \tilde{k}_{\mathrm{F}} \\
& +\frac{1}{24 \pi^{2}} \frac{1}{\widetilde{E}_{\mathrm{F}}}\left(\frac{\tilde{E}_{\mathrm{F}}}{\tilde{k}_{\mathrm{F}}}+\frac{\tilde{k}_{\mathrm{F}}}{\widetilde{E}_{\mathrm{F}}}+4 \frac{\tilde{M}^{2}}{\widetilde{E}_{\mathrm{F}}^{2}} \ln \frac{\tilde{k}_{\mathrm{F}}+\tilde{E}_{\mathrm{F}}}{\tilde{M}}\right)\left(\nabla \tilde{k}_{\mathrm{F}}\right)^{2} \\
& +\frac{1}{24 \pi^{2}} \frac{1}{\tilde{k}_{\mathrm{F}}} F_{i j} F^{i j}+\frac{1}{6 \pi^{2}} \frac{\tilde{M}}{\tilde{E}_{\mathrm{F}}} \ln \frac{\tilde{k}_{\mathrm{F}}+\widetilde{E}_{\mathrm{F}}}{\tilde{M}} \Delta \tilde{M} \\
& -\frac{1}{24 \pi^{2}} \frac{1}{\widetilde{E}_{\mathrm{F}}}\left(\frac{\tilde{k}_{\mathrm{F}}}{\widetilde{E}_{\mathrm{F}}}-4 \frac{\tilde{k}_{\mathrm{F}}^{2}}{\tilde{E}_{\mathrm{F}}^{2}} \ln \frac{\tilde{k}_{\mathrm{F}}+\tilde{E}_{\mathrm{F}}}{\tilde{M}}\right)(\nabla \tilde{M})^{2} \\
& -\frac{1}{12 \pi^{2}} \frac{1}{\tilde{M}}\left(1+\frac{\tilde{k}_{\mathrm{F}}^{2}}{\widetilde{E}_{\mathrm{F}}^{2}}+4 \frac{\tilde{k}_{\mathrm{F}} \tilde{M}^{2}}{\widetilde{E}_{\mathrm{F}}^{3}} \ln \frac{\tilde{k}_{\mathrm{F}}+\tilde{E}_{\mathrm{F}}}{\tilde{M}}\right)\left(\underline{\nabla} \tilde{k}_{\mathrm{F}}\right) \cdot(\underline{\nabla} \tilde{M}) \\
& \alpha^{[2]}\left(\tilde{k}_{\mathrm{F}}, \tilde{M}, j^{k}\right)=\alpha_{\mathrm{R}}^{[2]}\left(\tilde{k}_{\mathrm{F}}, \tilde{M}, j^{k}\right)+\alpha_{\mathrm{D}}^{[2]}\left(\tilde{k}_{\mathrm{F}}, \tilde{M}, j^{k}\right) \\
& \alpha_{\mathrm{R}}^{[2]}\left(\tilde{k}_{\mathrm{F}}, \tilde{M}, j^{k}\right)=-\frac{1}{6 \pi^{2}} \frac{\tilde{M} \tilde{k}_{\mathrm{F}}}{\tilde{E}_{\mathrm{F}}^{2}} \ln \left|\frac{\tilde{M}}{M}\right| \Delta \tilde{k}_{\mathrm{F}} \\
& +\frac{1}{12 \pi^{2}} \frac{1}{\tilde{M}}\left(\frac{\tilde{k}_{\mathrm{F}}^{2}}{\widetilde{E}_{\mathrm{F}}^{2}}-2 \frac{\tilde{M}^{4}}{\widetilde{E}_{\mathrm{F}}^{4}} \ln \left|\frac{\tilde{M}}{M}\right|\right)\left(\nabla \tilde{k}_{\mathrm{F}}\right)^{2} \\
& \left.-\frac{1}{24 \pi^{2}} \frac{1}{\tilde{M}} F_{i j} F^{i j}+\frac{1}{12 \pi^{2}}\left(1+2 \frac{\tilde{k}_{\mathrm{F}}^{2}}{\widetilde{E}_{\mathrm{F}}^{2}}\right) \ln \left|\frac{\tilde{M}}{M}\right|\right) \Delta \tilde{M} \\
& +\frac{1}{24 \pi^{2}} \frac{1}{\widetilde{M}}\left(1+2 \frac{\tilde{k}_{\mathrm{F}}^{2}}{\widetilde{E}_{\mathrm{F}}^{2}}-4 \frac{\tilde{k}_{\mathrm{F}}^{2} \tilde{M}^{2}}{\widetilde{E}_{\mathrm{F}}^{4}} \ln \left|\frac{\tilde{M}}{M}\right|\right)(\nabla \tilde{M})^{2} \\
& +\frac{1}{3 \pi^{2}} \frac{\tilde{k}_{\mathrm{F}} \tilde{M}^{2}}{\widetilde{E}_{\mathrm{F}}^{4}} \ln \left|\frac{\tilde{M}}{\bar{M}}\right|\left(\underline{\nabla} \tilde{k}_{\mathrm{F}}\right) \cdot(\underline{\nabla} \tilde{M})
\end{aligned}
$$




$$
\begin{aligned}
& \alpha_{\mathrm{D}}^{[2]}\left(\tilde{k}_{\mathrm{F}}, \tilde{M}, j^{k}\right)=-\frac{1}{6 \pi^{2}} \frac{\tilde{M} \tilde{k}_{\mathrm{F}}}{\tilde{E}_{\mathrm{F}}^{2}} \ln \frac{\tilde{k}_{\mathrm{F}}+\tilde{E}_{\mathrm{F}}}{\tilde{M}} \Delta \tilde{k}_{\mathrm{F}} \\
& +\frac{1}{24 \pi^{2}} \frac{1}{\tilde{M}}\left(3 \frac{\tilde{k}_{\mathrm{F}}^{3}}{\widetilde{E}_{\mathrm{F}}^{3}}-5 \frac{\widetilde{k}_{\mathrm{F}}}{\widetilde{E}_{\mathrm{F}}}-4 \frac{\tilde{M}^{4}}{\widetilde{E}_{\mathrm{F}}^{4}} \ln \frac{\widetilde{k}_{\mathrm{F}}+\widetilde{E}_{\mathrm{F}}}{\tilde{M}}\right)\left(\nabla \tilde{k}_{\mathrm{F}}\right)^{2} \\
& +\frac{1}{24 \pi^{2}} \frac{1}{\tilde{M}} \frac{\widetilde{k}_{\mathrm{F}}}{\widetilde{E}_{\mathrm{F}}} F_{i j} F^{i j}-\frac{1}{12 \pi^{2}}\left(\frac{\tilde{k}_{\mathrm{F}}}{\widetilde{E}_{\mathrm{F}}}-\left(1+2 \frac{\widetilde{k}_{\mathrm{F}}^{2}}{\tilde{E}_{\mathrm{F}}^{2}}\right) \ln \frac{\widetilde{k}_{\mathrm{F}}+\widetilde{E}_{\mathrm{F}}}{\tilde{M}}\right) \Delta \tilde{M} \\
& -\frac{1}{24 \pi^{2}} \frac{1}{\tilde{M}}\left(3 \frac{\tilde{k}_{\mathrm{F}}^{3}}{\widetilde{E}_{\mathrm{F}}^{3}}+4 \frac{\tilde{k}_{\mathrm{F}}^{2} \tilde{M}^{2}}{\widetilde{E}_{\mathrm{F}}^{4}} \ln \frac{\tilde{k}_{\mathrm{F}}+\tilde{E}_{\mathrm{F}}}{\tilde{M}}\right)(\nabla \tilde{M})^{2} \\
& +\frac{1}{12 \pi^{2}} \frac{1}{\widetilde{E}_{\mathrm{F}}}\left(3 \frac{\tilde{k}_{\mathrm{F}}^{2}}{\widetilde{E}_{\mathrm{F}}^{2}}+4 \frac{\tilde{k}_{\mathrm{F}} \tilde{M}^{2}}{\widetilde{E}_{\mathrm{F}}^{3}} \ln \frac{\tilde{k}_{\mathrm{F}}+\widetilde{E}_{\mathrm{F}}}{\tilde{M}}\right)\left(\nabla \tilde{k}_{\mathrm{F}}\right) \cdot(\nabla \tilde{M}) .
\end{aligned}
$$

Current conservation, $\partial_{k} j^{k}=0$, need not to be imposed explicitly as it is automatically generated, which can be seen by taking the divergence of Eq. (4.15).

Neglect of all second order contributions leads back to the well known TF approximation of QHD

$$
\tilde{M}=M-g_{\mathrm{s}} \chi, \quad \tilde{E}_{\mathrm{F}}+g_{\mathrm{v}} U_{0}=\mu, \quad g_{\mathrm{v}} U^{k}=0 .
$$

To this order $M^{*}$ is identical to $\tilde{M}$ and the vector current contribution vanishes, as the $h=0$ limit is equivalent to a homogeneous, rotational invariant system.

\section{Concluding Remarks}

Our final result (4.8), the ETF approximation to QHD, including second order gradient corrections to the kinetic energy of the baryons and Hartree terms corresponding to the exchange of scalar and vector mesons, constitutes a direct extension of the TF limit of the Hartree approximation to this model. New aspects of this functional are the consistent inclusion of radiative corrections as well as current contributions. Furthermore, our final form of the energy functional is fully inverted, i.e., the semiclassical quantities utilized for the derivation of this functional are eliminated completely, in contrast to previous semi-inverted versions [7].

We also have indicated how this approximation can be improved by using an exact representation of the noninteracting kinetic energy functional in the form of Kohn -Sham equations and including exchange-correlation contributions. It should be emphasized once again that the main advantage of the DFT approach in either the direct variational or the Kohn-Sham version is the systematic combination of inhomogeneity and interaction effects. Thus it might prove to be a very adequate tool for the discussion of finite strongly interacting systems.

In addition we have indicated an alternative to the standard normalisation conditions for the renormalisation of baryon loops, whose significance, however, remains to be established by a numerical investigation. This work is in progress. 
Obviously there is sufficient scope for extensions.

1. For the description of realistic nuclei the simple version of QHD considered in this work, i.e., QHD-I, has to be extended to include photon and isovector $(\rho$-meson) exchange in order to account for Coulomb effects and the neutron excess in heavy nuclei. This task is rather straightforward at the level of second order gradient corrections though more tedious.

The inclusion of scalar meson selfinteraction contributions which were shown to be necessary for a quantitative description of finite nuclei [29] would also not present any difficulties. The formalism developped in Section 2 is based on the fact that there is linear coupling between mesons and nucleons. It does not depend on the mesonic degrees of freedom. The internal structure of the mesonic part of the Lagrangian only enters into the explicit form of the Hartree terms, Eq. (2.33), and the exchange-correlation functional, Eq. (2.34). Nonlinear meson selfinteractions can be included by replacing the noninteracting scalar meson propagator by the propagator of, e.g., the $\phi^{4}$ theory, so that the Helmholtz equation (4.13) for the scalar meson is changed to

$$
\left(\Delta-m_{\mathrm{s}}^{2}\right) \chi(\underline{x})=-g_{\mathrm{s}} \rho_{\mathrm{s}}(\underline{x})+\frac{\kappa}{2 !} \chi^{2}(\underline{x})+\frac{\lambda}{3 !} \chi^{3}(\underline{x})
$$

in the ETF approach.

2. In order to approach the accuracy of Hartree-Fock results for finite nuclei within the variational approach to QHD one needs to include exchange contributions. In lowest order this would, together with the ETF functional presented here, constitute the Thomas Fermi-Dirac-Weizsäcker (TFDW) approximation to QHD. As in the nonrelativistic case sufficiently accurate groundstate properties of nuclei are only obtained with a gradient expansion of the kinetic energy to fourth order, extensions of the TFDW limit (in particular for the kinetic energy but also for the exchange contribution) are probably necessary. For the kinetic energy such an extension is possible (though rather cumbersome) with the methods outlined in Section 3.

3. In order to go beyond the Hartree-Fock approximation for either the variational or the Kohn-Sham approach an investigation of correlation contributions will be necessary. In part one may follow here the standard approach to transcribe the results of the homogeneous limit to the inhomogeneous case in order to obtain the local density approximation for these terms, using, e.g., the results of Chin [8]. A more direct investigation of the relative importance of ladder versus ring contributions for the homogeneous case could constitute the starting point for the discussion of correlation contributions.

4. Especially for applications of QHD in heavy-ion collisions and astrophysics the theory has to be formulated for the case of finite temperature. An ETF formulation for $T \neq 0$ has recently been given for the case of QED [30]. The generalisation to QHD is under way [31]. 


\section{APPENDIX}

The lowest order contribution of the propagator $g(x, p)$, Eq. (3.13), can be represented in several equivalent forms. Starting with

$$
g^{[0]}(\underline{x}, p)=\left(\not p+M^{*}\right) \tilde{g}^{[0]}(\underline{x}, p),
$$

where

$$
\tilde{g}^{[0]}(\underline{x}, p)=\frac{1}{p^{2}-M^{* 2}+i \varepsilon}+2 \pi i \delta\left(p^{2}-M^{* 2}\right) \theta\left(p_{0}\right) \theta\left(E_{\mathrm{F}}^{*}-p_{0}\right),
$$

one can express the product

$$
2 \pi i \delta\left(p^{2}-M^{* 2}\right) \theta\left(p_{0}\right)
$$

in the valence nucleon part by the difference of two pole contributions. This leads to

$$
\tilde{g}^{[0]}(\underline{x}, p)=\frac{1}{p_{0}^{2}-\left(E^{*}-i \varepsilon\right)^{2}}+\left\{\frac{1}{\left(p_{0}-i \varepsilon\right)^{2}-E^{* 2}}-\frac{1}{p_{0}^{2}-\left(E^{*}-i \varepsilon\right)^{2}}\right\} \theta\left(E_{\mathrm{F}}^{*}-p_{0}\right)
$$

which may be factorised as

$$
\tilde{g}^{[0]}(x, p)=\frac{1}{p_{0}^{2}-\left(E^{*}-i \varepsilon\right)^{2}}+\frac{\theta\left(E_{\mathrm{F}}^{*}-p_{0}\right)}{p_{0}+E^{*}-i \varepsilon}\left\{\frac{1}{p_{0}-E^{*}-i \varepsilon}-\frac{1}{p_{0}-E^{*}+i \varepsilon}\right\} .
$$

The difference of the simpler pole contributions can be reexpressed by a $\delta$ function and the shift in the pole at $p_{0}=-E^{*}$ may be dropped as only $p_{0}=E^{*}$ contributes

$$
\begin{aligned}
\tilde{g}^{[0]}(x, p) & =\frac{1}{p_{0}^{2}-\left(E^{*}-i \varepsilon\right)^{2}}+2 \pi i \frac{\delta\left(p_{0}-E^{*}\right)}{p_{0}+E^{*}} \theta\left(E_{F}^{*}-p_{0}\right) \\
& =\frac{1}{p_{0}^{2}-\left(E^{*}-i \varepsilon\right)^{2}}+2 \pi i \frac{\delta\left(p_{0}-E^{*}\right)}{2 E^{*}} \theta\left(E_{\mathrm{F}}^{*}-p_{0}\right) .
\end{aligned}
$$

The product of $n$ zero order propagators is most easily expressed, using the representation (A.3), as

$$
\begin{aligned}
{\left[\tilde{g}^{[0]}(\underline{x}, p)\right]^{n}=} & \frac{1}{\left[p_{0}^{2}-\left(E^{*}-i \varepsilon\right)^{2}\right]^{n}} \\
& +\left\{\frac{1}{\left[\left(p_{0}-i \varepsilon\right)^{2}-E^{* 2}\right]^{n}}-\frac{1}{\left[p_{0}^{2}-\left(E^{*}-i \varepsilon\right)^{2}\right]^{n}}\right\} \theta\left(E_{\mathrm{F}}^{*}-p_{0}\right) .
\end{aligned}
$$


The same steps leading from Eq. (A.3) to Eq. (A.4) can be repeated to obtain

$$
\left[\tilde{g}^{[0]}(\underline{x}, p)\right]^{n}=\frac{1}{\left[p_{0}^{2}-\left(E^{*}-i \varepsilon\right)^{2}\right]^{n}}+2 \pi i \frac{(-1)^{n-1}}{(n-1) !} \frac{\delta^{(n-1)}\left(p_{0}-E^{*}\right)}{\left(p_{0}+E^{*}\right)^{n}} \theta\left(E_{\mathrm{F}}^{*}-p_{0}\right)
$$

Introducing the identity

$$
\delta^{(n-1)}\left(p_{0}-E^{*}\right)=\left(p_{0}+E^{*}\right)^{n} \delta^{(n-1)}\left(p_{0}^{2}-E^{* 2}\right)
$$

in Eq. (A.6) one finds the alternative representation

$$
\begin{aligned}
& {\left[\tilde{g}^{[0]}(\underline{x}, p)\right]^{n}} \\
& \quad=\frac{1}{\left[p_{0}^{2}-\left(E^{*}-i \varepsilon\right)^{2}\right]^{n}}+2 \pi i \frac{(-1)^{n-1}}{(n-1) !} \delta^{(n-1)}\left(p_{0}^{2}-E^{* 2}\right) \theta\left(p_{0}\right) \theta\left(E_{\mathrm{F}}^{*}-p_{0}\right),
\end{aligned}
$$

where an additional factor $\theta\left(p_{0}\right)$ needs to be included in order to eliminate the contributions from $p_{0}=-E^{*}$. For $n=2$ representation (A.7) reduces to the result given by Chin [8]. The representation (A.6) is, however, preferable as mathematical ambiguities are avoided.

\section{ACKNOWLEDGMENTS}

We thank H. Müller and Professor S. H. Vosko for very valuable discussions. This work has been supported in part by the BMFT (Projekt der Verbundforschung 06 OF 705) and the Natural Sciences and Engineering Research Council of Canada (Grant OGP 6294).

Note added in proof. After this manuscript was submitted there appeared a contribution by Weigel, Haddad, and Weber [32], which adresses a similar problem from a different angle. The authors use the Hartree-Fock approximation to QHD (including $\sigma-, \omega-, \rho_{-}, \pi$ - and photon exchange) as a starting point and derive a semiclassical expansion of the Wigner transformed Green's function and the baryonic density on the basis of the Wigner-Kirkwood expansion. Recently, an extension of this work to finite temperatures has been published [33].

\section{REFERENCES}

1. R. M. Dreizler and E. K. U. Gross, "Density Functional Theory," Springer-Verlag, Berlin, 1990; R. G. Parr AND W. YANG, "Density Functional Theory of Atoms and Molecules," Oxford Univ. Press, New York, 1989; E. V. LudeÑA AND E. S. KryachKo, "Energy Density Functional Theory of Many-Electron Systems," Kluwer, Dordrecht, 1990; R. O. Jones and O. Gunnarsson, Rev. Mod. Phys. 61 (1989), 689.

2. M. Brack, C. Guet, and H. B. Hakansson, Phys. Rep. 123 (1985), 275; M. Pi, X. Viñas, F. Garcias, and M. Barranco, Phys. Lett. B 215 (1988), 5; M. Centelles, M. Pi, X. Viñas, F. Garcias, and M. Barranco, Nucl. Phys. A 510 (1990), 397.

3. J. D. Walecka, Ann. Phys. (N.Y.) 83 (1974), 491. 
4. J. Bogula And A. R. Bodmer, Nucl. Phys. A 292 (1977), 413.

5. B. D. Serot, Phys. Lett. B 86 (1979), 146; Phys. Lett. B 87 (1979), 403.

6. J. Boguta and J. Rafelski, Phys. Lett. $B 71$ (1977), 22; F. E. Serr and J. D. Walecka. Phys. Lett. B 79 (1978), 10; Phys. Lett. B 84 (1979), 529.

7. M. Centelles, X. Viñas, M. Barranco, and P. Schuck, Nucl. Phys. a 519 (1990), 73c.

8. S. A. Chin, Ann. Phys. (N.Y.) 108 (1977), 301.

9. C. J. Horrowirz AND B. D. SERot, Phys. Lett. B $140(1984), 181$.

10. R. J. PERry, Phys. Lett. B $182(1986), 269$.

11. D. A. Wasson, Phys. Lett. B 210 (1988), 41.

12. C. E. Price and G. E. Walker, Phys. Rev. C 36 (1987), 354; W. Pannert, P. Ring, And J. Boguta, Phys. Rev. Lett. 59 (1987), 2420; S. J. LeE, J. Fink, A. B. Balantekin, M. R. Strayer, A. S. Umar, P. G. Rejnhardt, J. A. Maruhn, and W. Greiner, Phys. Rev. Lett. 57 (1986), 2916; Phys. Rev. Lett. 59 (1987), 1171; W. KoepF And P. Rung, Phys. Lett. B 212 (1988), 397.

13. U. Hofmann and P. Ring, Phys. Lett. B 214 (1988), 307.

14. H. MÜller, E. Engel, And R. M. Dreizler, Phys. Rev. A 40 (1989), 5542; E. Engel And R. M. Dreizler, Phys, Rev. A 35 (1987), 3607.

15. B. D. Serot And J. D. WaleckA, Adv. Nucl. Phys. 16 (1985), 1.

16. P. Hohenberg and W. Kohn, Phys. Rev. B 136 (1964), 864.

17. W. Kohn and L. J. Sham, Phys, Rev. A 140 (1965), 1133.

18. H. P. DürR, Phys. Rev. 103 (1956), 469.

19. A. K. Rajagopal and J. Callaway, Phys. Rev. B 7 (1973), 1912; A. K. Rajagopal, J. Phys. C 11 (1978), L943.

20. A. H. MacDonald and S. H. Vosko, J. Phys. C 12 (1979), 2977.

21. E. ENGEL AND S. H. VosKo, to be submitted for publication.

22. G. Vignale and M. Rasolt, Phys, Rev. Lett, 59 (1987), 2360; G. Vignale and M. Rasolt, Phys. Rev. B 37 (1988), 10685 .

23. L. Levy, Proc. Natl. Acad. Sci. U.S.A. 76 (1979), 6062; E. H. Lieb, Int. J. Quant. Chem. 24 (1983), 243; E. H. Lieb, Rev. Mod. Phys. 53 (1981), 603.

24. E. Engel, H. Mülifr and R. M. Dreizliter, Phys. Rev. A 39 (1989), 4873.

25. C. ITZYKSON AND J. B. Zuber, "Quantum Field Theory," McGraw-Hill, New York, 1980.

26. B. K. Jennings, Phys. Lett. B 74 (1978), 13.

27. G. "T Hooft and M. Veltman, Nucl. Phys. B 44 (1972), 189.

28. B. Grammaticos and A. Voros, Ann. Phys. (N.Y.) 123 (1979), 359; 129 (1980), 153.

29. P. G. Reinhardt, M. Rufa, J. Maruhn, W. Greiner, and J. Friedrich, Z. Phy:s. A 323 (1986), 13.

30. H. J. A. C. Stroucken and R. M. Dreizler, Phys. Rev. A 43 (1991), 3401.

31. H. MÜLLER AND R, M. DREIZLER, submitted for publication.

32. M. K. Weigel, S. Haddad, and F. Weber, J. Phys. G 17 (1991), 619.

33. D. Von-Eiff and M. K. Weigel, Z. Phys. A 339 (1991), 63. 